\title{
Mortality Forecasting for Multiple Populations: An Augmented Common Factor Model with a Penalized Log-Likelihood
}

\author{
Mao Kang*, Yanxin Liu† Johnny Siu-Hang Li $^{\ddagger}$ and Wai-Sum Chan ${ }^{\S}$
}

\begin{abstract}
Recently, the topic of multi-population mortality forecasting has gained considerable attention among researchers and end-users. One of the most popular multi-population mortality models is the augmented common factor (ACF) model. In spite of its popularity, the ACF model is subject to the limitation of producing mortality forecasts with a jagged pattern rather than a smooth relationship with age. In this paper, we attempt to mitigate this problem by generalizing the work of Delwarde et al. (2007) to a multi-population setting. The generalization involves $\left(n_{p}+1\right)$ smoothing parameters, one for the common trend and one for each of the $n_{p}$ individual populations. The smoothing parameters are determined by using an extended leave-one-out cross-validation. We illustrate the proposed extension with real mortality data. It is found that compared to the original ACF model, the proposed extension produces mortality forecasts that are more reasonable, with less jagged patterns across ages.
\end{abstract}

Key words: Demographic forecasting, Mortality forecasting, Parameter smoothing, Stochastic modeling, The Lee-Carter model

\section{Introduction}

Stochastic mortality models have a wide range of practical applications. For instance, governments rely on projections made from such models to engineer social security systems and demographic policies, while insurance companies depend on such models to quantify the value of and the risk associated with life-contingent liabilities. Compared to deterministic approaches that typically yield only a single estimate, stochastic mortality models produce also probabilistic confidence intervals and sample paths, which allow the user to gauge the degree of uncertainty involved and to understand how mortality rates may possibly evolve from now to a future time point.

\footnotetext{
${ }^{*}$ University of Waterloo

${ }^{\dagger}$ University of Nebraska-Lincoln

${ }^{\ddagger}$ University of Waterloo and The University of Melbourne

$\S$ The Chinese University of Hong Kong
} 
In recent years, stochastic mortality models for modeling multiple populations have gained considerable attention among researchers and end-users (see, e.g., Li and Hardy, 2011; Ahmadi and Li, 2014; Kleinow, 2015). Multi-population mortality models can be used to ensure consistency of a national mortality projection with the mortality experiences observed in neighbouring countries that possess similar demographic characteristics (see Wan and Bertschi, 2015). By incorporating data from a larger population, multi-population mortality models may also be used to enhance the credibility of the mortality projections for small populations (e.g., a pension plan of a small firm) with thin volumes of death and exposure counts (see Plat, 2009). Multi-population mortality models also permit us to project future mortality of both genders jointly (see Li, 2013). The resulting projections are particularly helpful to actuaries working in jurisdictions where genderneutral pricing is enforced.

Further, multi-population mortality models are crucially important in mortality and longevity risk securitizations. Since about 10 years ago, capital market derivatives that are linked to future mortality experience have been made available to insurance companies and pension plan sponsors as a risk management tool. Users of mortality-linked derivatives are subject to some residual basis risk, because the derivatives are typically associated with national populations, which can be considerably different from the users' own populations of individuals. Although cannot be eliminated, this piece of residual risk can be quantified by using a multi-population mortality model that is estimated to data from the populations involved (see Chan et al., 2016; Li et al., 2017, 2018; Liu and Li, 2016). Multi-population mortality models also enable market participants to analyze sophisticated mortality-linked securities that are associated with more than one population. A prime example is Swiss Re's Kortis bond, whose payoff is linked to the difference in the mortality improvements experienced between English and Welsh males and US males (see Hunt and Blake, 2015; Li et al., 2017).

Researchers have recently introduced a number of multi-population stochastic mortality models. Among these models, the augmented common factor (ACF) model introduced by Li and Lee (2005) has been considered very often in the literature (see, e.g., Li, 2013; Li and Hardy, 2011), possibly because it can be regarded as a generalization of the renowned Lee-Carter model (Lee and Carter, 1992), which has already gained acceptance in the academic community (see, e.g., Booth, 2006; Booth et al., 2005, 2006). The ACF model is meticulously constructed so that the resulting forecasts are coherent, in the sense that the projected mortality trends for different populations do not diverge indefinitely over time. In spite of its popularity and the property of coherence it possesses, the ACF model is subject to a significant limitation: owing to its semi-parametric nature, it may result in forecasted mortality rates that exhibit a jagged pattern rather than a smooth relationship with age.

In more detail, the unwanted jaggedness arises from the erratic variations in the estimates of parameters representing the age pattern of mortality improvement. These irregularities typically carry no demographic meaning, which means that they are likely consequences of sampling errors. A mortality projection with a jagged pattern across ages appears to be anti-intuitive, because it 
is difficult to justify, for example, why mortality rates at adjacent ages change at very different speeds. It also causes problems in actuarial work, because a life table with a jagged pattern may lead to unreasonable pricing results. The need for a smooth projected life table is discussed further by Renshaw and Haberman (2003).

Methods for removing erratic variations in mortality projections can be divided into two categories: (i) pre-estimation and (ii) within-estimation. A prime example of pre-estimation methods is the Hyndman-Ullah approach (Hyndman and Ullah, 2007), in which crude death rates are first smoothed by weighted penalized regression splines. The collection of the smoothed rates (which are assumed to be the underlying, unobserved death rates) is then modeled by a basis function expansion with one or more principal components. The Hyndman-Ullah approach has been generalized to a multi-population version called the product-ratio approach (Hyndman et al., 2013), which produces smoothed coherent mortality forecasts for related sub-populations.

However, as Delwarde et al. (2007) pointed out, pre-estimation methods and rather ad hoc and may lead to significant inconsistencies between observed values and model predictions. Because smoothing and estimation are performed in different stages, the distinction between observational variance and the variation among the underlying mortality rates may be sub-optimal. If crude mortality rates are over-smoothed in the first stage, then the resulting model may not adequately capture the true age patterns of mortality and changes in mortality. To avoid this problem, one may use within-estimation methods, in which parameter smoothing and estimation are performed in one single stage. For example, Delwarde et al. (2007) propose fitting the Lee-Carter model (Lee and Carter, 1992) with a penalized least squares or log-likelihood method, whereby an optimized smoothing parameter is used to strike the best balance between goodness-of-fit and smoothness. Another example is the smooth Lee-Carter model developed by De Jong and Tickle (2006), which produces smooth mortality forecasts by using a design matrix that reduces the dimension of the original model.

The mentioned within-estimation methods are applicable to single-population mortality models only. In this paper, we attempt to generalize the work of Delwarde et al. (2007) to a multipopulation setting that is compatible with the ACF model for $n_{p} \geq 2$ populations. The generalization involves $\left(n_{p}+1\right)$ smoothing parameters, one for the common trend and one for each of the $n_{p}$ individual populations. The optimized smoothing parameters are identified by using a leaveone-out cross-validation, which is more sophisticated than that entailed in the single-population setting due to the increased number of smoothing parameters. We acknowledge that smooth coherent multi-population mortality forecasts may also be generated by other methods, including the coherent generalized linear modeling approach (Ahmadi and Li, 2014) and parallel P-splines regressions (Currie et al., 2004, Section 4.2), but these methods are not able to provide sample paths (trajectories) of future mortality rates, which are useful in many actuarial applications including Value-at-Risk and hedge ratio calculations (see, e.g., Li and Hardy, 2011; Zhou and Li, 2017). 
The rest of this paper is organized as follows. Section 2 reviews the methodology proposed by Delwarde et al. (2007). Section 3 presents our generalization for modeling multiple populations. Section 4 applies the proposed generalization to real mortality data from a pair of populations. Section 5 compares our proposed generalization with the original ACF model and the productratio approach. Finally, Section 6 concludes the paper.

\section{A Review of the Single-Population Set-up}

We let $m_{x, t}$ be the central death rate at age $x$ and in year $t$. The original, single-population Lee-Carter model can be expressed as

$$
\ln \left(m_{x, t}\right)=a_{x}+b_{x} k_{t}+\varepsilon_{x, t}
$$

where $a_{x}$ is an age-specific parameter representing the average level of mortality at age $x, k_{t}$ is a time-varying mortality index indicating the overall level of mortality in year $t, b_{x}$ is another age-specific parameter that measures the sensitivity of the mortality at age $x$ to the time-varying mortality index, and $\varepsilon_{x, t}$ is the error term.

To generate forecasts of future mortality, the time-varying mortality index $k_{t}$ is further modeled by a random walk with drift:

$$
k_{t}=\delta+k_{t-1}+\xi_{t}
$$

where $\delta$ is a constant and $\left\{\xi_{t}\right\}$ is a sequence of i.i.d. normal random variables with a zero mean and a constant variance.

The original Lee-Carter model typically yields mortality forecasts that exhibit a jagged pattern across ages. This problem is due primarily to the sampling variation in the raw estimates of $b_{x}$. To overcome this problem, Delwarde et al. (2007) proposed two estimation methods which ensure that the resulting estimates of $b_{x}$ are to a certain extent smooth across ages.

The first method is penalized least squares, in which the Lee-Carter model parameters are obtained by minimizing the following objective function:

$$
\sum_{x=x_{\min }}^{x_{\max }} \sum_{t=t_{\min }}^{t_{\max }}\left(\ln \left(m_{x, t}\right)-a_{x}-b_{x} k_{t}\right)^{2}+\vec{b}^{\prime} \mathbf{P} \vec{b}
$$

where $\left[x_{\min }, x_{\max }\right]$ represents the age range under consideration, $\left[t_{\min }, t_{\max }\right]$ denotes the calibration window to which the model is estimated, $\vec{b}=\left(b_{x_{\min }}, \ldots, b_{x_{\max }}\right)^{\prime}$ and $\mathbf{P}$ is the penalty matrix. In particular, $\mathbf{P}$ is given by

$$
\mathbf{P}=\pi \boldsymbol{\Delta}^{\prime} \boldsymbol{\Delta}
$$


where $\pi$ is a pre-selected smoothing parameter and

$$
\boldsymbol{\Delta}=\left(\begin{array}{cccccc}
1 & -2 & 1 & 0 & \ldots & 0 \\
0 & \ddots & \ddots & \ddots & \ddots & \vdots \\
\vdots & \ddots & \ddots & \ddots & \ddots & \vdots \\
0 & \ldots & 0 & 1 & -2 & 1
\end{array}\right)
$$

is a $\left(x_{\max }-x_{\min }-1\right) \times\left(x_{\max }-x_{\min }+1\right)$ band matrix.

The second method is penalized log-likelihood. In contrast to the previous method which requires no distributional assumption, this method assumes that

$$
D_{x, t} \sim \operatorname{Poisson}\left(E_{x, t} \exp \left(a_{x}+b_{x} k_{t}\right)\right)
$$

where $D_{x, t}$ denotes the (random) death count at age $x$ and in year $t$, and $E_{x, t}$ represents the corresponding (non-random) exposure count. The Lee-Carter model parameters are obtained by maximizing the penalized log-likelihood, which is given by

$$
\sum_{x=x_{\min }}^{x_{\max }} \sum_{t=t_{\min }}^{t_{\max }}\left(d_{x, t}\left(a_{x}+b_{x} k_{t}\right)-E_{x, t} \exp \left(a_{x}+b_{x} k_{t}\right)\right)-\frac{1}{2} \vec{b}^{\prime} \mathbf{P} \vec{b}
$$

where $d_{x, t}$ is the realized value of $D_{x, t}$ and the penalty matrix $\mathbf{P}$ is the same as that specified by equation (2.1). In this expression, the first term (the summand) represents the model's loglikelihood (excluding the constant term that does not depend on the parameters) while the second term represents the roughness penalty.

In both methods, the degree of smoothness in the estimates of $b_{x}$ across ages is determined by the value of $\pi$. When $\pi=0$, the parameter estimates are not smoothed at all and are identical to the ordinary least squares estimates (if the first method is used) or maximum likelihood estimates (if the second method is used). In the other extreme when $\pi \rightarrow \infty$, the estimates of $b_{x_{\min }}, \ldots, b_{x_{\max }}$ lie on a straight line perfectly. The value of $\pi$ is selected by a leave-one-out cross-validation, which involves the following steps:

(i) Given a value of $\pi$, re-estimate the Lee-Carter parameters to a pseudo data set with one observation being intentionally left out; use the re-estimated model to 'predict' the corresponding missing observation; record the 'prediction error'.

(ii) Repeat step (i) by leaving out one other observation at a time until all observations in the full data set have been considered.

(iii) Repeat steps (i) and (ii) for different possible values of $\pi$; choose the value of $\pi$ that leads to the smallest sum of squared prediction errors. 


\section{A Generalization to Incorporate Multiple Populations}

\subsection{The ACF Model}

Li and Lee (2005) generalized the original Lee-Carter model to a multi-population version, which they call the augmented common factor (ACF) model. Suppose that $n_{p} \geq 2$ populations are being modeled. We let $m_{x, t, i}$, where $i=1, \ldots, n_{p}$, be population $i$ 's central rate of death at age $x$ and in year $t$. The ACF model describes the logarithm of $m_{x, t, i}$ as follows:

$$
\ln \left(m_{x, t, i}\right)=a_{x, i}+B_{x} K_{t}+b_{x, i} k_{t, i}+\varepsilon_{x, t, i}, \quad i=1, \ldots, n_{p},
$$

where $a_{x, i}$ is a parameter measuring population $i$ 's average level of mortality at age $x, K_{t}$ is a time-varying index reflecting the overall mortality level of all populations being modeled at time $t, k_{t, i}$ is another time-varying index that applies to population $i$ only, $B_{x}$ and $b_{x, i}$ respectively measure the sensitivities of $\ln \left(m_{x, t, i}\right)$ to changes in $K_{t}$ and $k_{t, i}$, and $\varepsilon_{x, t, i}$ is the error term that captures all remaining variations.

To generate forecasts of future mortality, $K_{t}$ is further modeled by a random walk with drift,

$$
K_{t}=\delta_{c}+K_{t-1}+\xi_{t},
$$

while $k_{t, i}$ is further modeled by a first order autoregression,

$$
k_{t, i}=\phi_{0, i}+\phi_{1, i} k_{t-1, i}+\xi_{t, i}, \quad i=1, \ldots, n_{p},
$$

where $\delta_{c}$ and $\phi_{0, i}$ are constants, $\phi_{1, i}$ is another constant whose absolute value is strictly less than 1 , and $\left\{\xi_{t}\right\}$ and $\left\{\xi_{t, i}\right\}$ for $i=1, \ldots, n_{p}$ are sequences of i.i.d. normal random variables with zero mean and constant variances.

In the ACF model, parameters $B_{x}$ and $K_{t}$ capture the common mortality trend among the group of populations being modeled, while parameters $b_{x, i}$ and $k_{t, i}$ permit the mortality trend for population $i$ to deviate from the common trend. The use of a first order autoregression for $k_{t, i}$ ensures that the projected mortality trend for population $i$ does not diverge indefinitely from the common trend over time. The resulting mortality projections are therefore regarded as coherent.

In the original work of $\mathrm{Li}$ and Lee (2005), the ACF model is estimated in multiple stages. First, the value of $a_{x, i}$ is set to the average of $\ln \left(m_{x, t, i}\right)$ over $t=t_{\min }, \ldots, t_{\max }$. Second, parameters $B_{x}$ and $K_{t}$ are estimated by applying a first order singular value decomposition (SVD) to the

matrix of $\sum_{i=1}^{n_{p}} w_{i}\left(\ln \left(m_{x, t, i}\right)-\hat{a}_{x, i}\right)$, where $w_{i}$ is the weight on the $i$ th population and the ‘`' sign indicates that the value underneath is an estimate. The weights $w_{1}, \ldots, w_{n_{p}}$ may be determined using the relative population sizes. Finally, parameters $b_{x, i}$ and $k_{t, i}$ are estimated by applying a first order SVD to the matrix of $\ln \left(m_{x, t, i}\right)-\hat{a}_{x, i}-\hat{B}_{x} \hat{K}_{t}$.

One problem of this multi-stage estimation method is that it does not guarantee the resulting parameter estimates are optimal. In particular, they generally neither minimize the sum of squared 
errors nor maximize the model's log-likelihood. More importantly, the resulting estimates of $B_{x}$ and $b_{x, i}$ typically exhibit a certain degree of erratic variations, leading to mortality forecasts that are not smooth across ages. In what follows, we extend the two methods of Delwarde et al. (2007) to the ACF model, with an aim to produce smooth mortality forecasts for multiple populations simultaneously.

\subsection{Penalized Least Squares for the ACF Model}

Both the ACF model and the original Lee-Carter model can be considered as semi-parametric. In particular, rather than using smooth parametric age functions, the ACF model assigns two parameters $\left(B_{x}\right.$ and $\left.b_{x, i}\right)$ to each age $x$ to capture the response of the log mortality rate at age $x$ to changes in the common mortality index $\left(K_{t}\right)$ and the population-specific mortality index $\left(k_{t, i}\right)$. The use of a large number of parameters may lead to over-fitting, so that the age-response parameters $B_{x}$ and $b_{x, i}$ are sensitive to sampling errors and may consequently exhibit irregular patterns across ages.

To prevent over-fitting, we should not only optimize goodness-of-fit but also penalize the use of excessive model parameters. One way to achieve this goal is to use the method of penalized least squares. For the ACF model, the penalized least squares parameter estimates can be obtained by minimizing the objective function below:

$$
\sum_{i=1}^{n_{p}} \sum_{x=x_{\min }}^{x_{\max }} \sum_{t=t_{\min }}^{t_{\max }}\left(\ln m_{x, t, i}-a_{x, i}-B_{x} K_{t}-b_{x, i} k_{t, i}\right)^{2}+\vec{B}^{\prime} \mathbf{P}_{c} \vec{B}+\sum_{i=1}^{n_{p}} \vec{b}_{i}^{\prime} \mathbf{P}_{i} \vec{b}_{i}
$$

where $\vec{B}=\left(B_{x_{\min }}, \ldots, B_{x_{\max }}\right)^{\prime}$ and $\vec{b}_{i}=\left(b_{x_{\min }, i}, \ldots, b_{x_{\max }, i}\right)^{\prime}$.

The first term in the expression above measures the sum of squared errors produced by the estimated model. The second term, with

$$
\mathbf{P}_{c}=\pi_{c} \Delta^{\prime} \Delta
$$

and $\pi_{c}$ being a pre-selected smoothing parameter, represents the roughness penalty that is applied to the common age-response parameters $B_{x_{\min }}, \ldots, B_{x_{\max }}$. The last term, with

$$
\mathbf{P}_{i}=\pi_{i} \Delta^{\prime} \Delta
$$

and $\pi_{1}, \ldots, \pi_{n_{p}}$ being $n_{p}$ other pre-selected smoothing parameters, is the total roughness penalty for the population-specific age-response parameters $b_{x_{\min }, i}, \ldots, b_{x_{\max }, i}, i=1, \ldots, n_{p}$. In principle, a roughness penalty may also be applied to parameters $a_{x_{\min }, i}, \ldots, a_{x_{\max }, i}$ for $i=1, \ldots, n_{p}$. However, as a matter of empirical fact, the crude estimates of these parameters are typically smooth across ages and hence require no further smoothing.

Specified in equation $(2.2), \boldsymbol{\Delta}$ is a $\left(x_{\max }-x_{\min }-1\right) \times\left(x_{\max }-x_{\min }+1\right)$ band matrix. The specification of $\boldsymbol{\Delta}$ implies that a second degree polynomial is used as a standard of smoothness. 
Equivalently speaking, we determine the degree of roughness by using the sum of the squared second order differences of the model parameters.

There are in total $\left(n_{p}+1\right)$ smoothing parameters $\left(\pi_{c} ; \pi_{1}, \ldots, \pi_{n_{p}}\right)$, controlling the trade-off between smoothness and goodness-of-fit. The larger a smoothing parameter is, the smoother the corresponding age-response parameters are. In the extreme case when a smoothing parameter tends to infinity, the corresponding age-response parameters become constant across ages. In this paper, we propose to select the smoothing parameters with an extended leave-one-out crossvalidation, which is detailed in Section 3.4. However, one may also determine the smoothing parameters by other methods, including expert judgement.

Given a chosen collection of smoothing parameters, the parameters in the ACF model can be estimated by an iterative Newton's algorithm, which is derived below:

$$
\left\{\begin{aligned}
\mathbf{C}_{a_{i}}^{(j)} \vec{a}_{i}^{(j+1)} & =\mathbf{C}_{a_{i}}^{(j)} \vec{a}_{i}^{(j)}+\vec{r}_{a_{i}}^{(j)} \\
\mathbf{C}_{K}^{(j)} \vec{K}^{(j+1)} & =\mathbf{C}_{K}^{(j)} \vec{K}^{(j)}+\vec{r}_{K}^{(j)} \\
\left(\mathbf{C}_{B}^{(j)}+\mathbf{P}_{c}\right) \vec{B}^{(j+1)} & =\mathbf{C}_{B}^{(j)} \vec{B}^{(j)}+\vec{r}_{B}^{(j)} \\
\mathbf{C}_{k_{i}}^{(j)} \vec{k}_{i}^{(j+1)} & =\mathbf{C}_{k_{i}}^{(j)} \vec{k}_{i}^{(j)}+\vec{r}_{k_{i}}^{(j)} \\
\left(\mathbf{C}_{b_{i}}^{(j)}+\mathbf{P}_{i}\right) \vec{b}_{i}^{(j+1)} & =\mathbf{C}_{b_{i}}^{(j)} \vec{b}_{i}^{(j)}+\vec{r}_{b_{i}}^{(j)}
\end{aligned}\right.
$$

where $\vec{a}_{i}=\left(a_{x_{\min }, i}, \ldots, a_{x_{\max }, i}\right)^{\prime}, \vec{K}=\left(K_{t_{\min }}, \ldots, K_{t_{\max }}\right)^{\prime}, \vec{k}_{i}=\left(k_{t_{\min }, i}, \ldots, k_{t_{\max }, i}\right)^{\prime}$, and $\vec{a}_{i}^{(j)}, \vec{B}^{(j)}$, $\vec{b}_{i}^{(j)}, \vec{K}^{(j)}$ and $\vec{k}_{i}^{(j)}$ denote the estimates of $\vec{a}_{i}, \vec{B}, \vec{b}_{i}, \vec{K}$ and $\vec{k}_{i}$ in the $j$ th iteration, respectively.

In above collection of equations, $\mathbf{C}_{a_{i}}^{(j)}, \mathbf{C}_{B}^{(j)}$ and $\mathbf{C}_{b_{i}}^{(j)}$ are $\left(x_{\max }-x_{\min }+1\right) \times\left(x_{\max }-x_{\min }+1\right)$ diagonal matrices, whose $\left(x-x_{\min }+1, x-x_{\min }+1\right)$ th elements are respectively given by

$$
\begin{aligned}
& \left(\mathbf{C}_{a_{i}}^{(j)}\right)_{x-x_{\min }+1, x-x_{\min }+1}=t_{\max }-t_{\min }+1, \\
& \left(\mathbf{C}_{B}^{(j)}\right)_{x-x_{\min }+1, x-x_{\min }+1}=2 \sum_{t=t_{\min }}^{t_{\max }}\left(K_{t}^{(j)}\right)^{2}
\end{aligned}
$$

and

$$
\left(\mathbf{C}_{b_{i}}^{(j)}\right)_{x-x_{\min }+1, x-x_{\min }+1}=\sum_{t=t_{\min }}^{t_{\max }}\left(k_{t, i}^{(j)}\right)^{2},
$$

whereas $\mathbf{C}_{K}^{(j)}$ and $\mathbf{C}_{k_{i}}^{(j)}$ are $\left(t_{\max }-t_{\min }+1\right) \times\left(t_{\max }-t_{\min }+1\right)$ diagonal matrices, whose $\left(t-t_{\min }+\right.$ $\left.1, t-t_{\min }+1\right)$ th elements are respectively given by

$$
\left(\mathbf{C}_{K}^{(j)}\right)_{t-t_{\min }+1, t-t_{\min }+1}=2 \sum_{x=x_{\min }}^{x_{\max }}\left(B_{x}^{(j)}\right)^{2},
$$


and

$$
\left(\mathbf{C}_{k_{i}}^{(j)}\right)_{t-t_{\min }+1, t-t_{\min }+1}=\sum_{x=x_{\min }}^{x_{\max }}\left(b_{x, i}^{(j)}\right)^{2} .
$$

Further, $\vec{r}_{a_{i}}^{(j)}, \vec{r}_{B}^{(j)}$ and $\vec{r}_{b_{i}}^{(j)}$ are column vectors with a dimension of $\left(x_{\max }-x_{\min }+1\right)$, whose $\left(x-x_{\min }+1\right)$ th elements are respectively given by

$$
\begin{aligned}
& \left(\vec{r}_{a_{i}}^{(j)}\right)_{x-x_{\min }+1}=\sum_{t=t_{\min }}^{t_{\max }}\left(\ln \left(m_{x, t, i}\right)-a_{x, i}^{(j)}-B_{x}^{(j)} K_{t}^{(j)}-b_{x, i}^{(j)} k_{t, i}^{(j)}\right), \\
& \left(\vec{r}_{B}^{(j)}\right)_{x-x_{\min }+1}=\sum_{i=1}^{n_{p}} \sum_{t=t_{\min }}^{t_{\max }} K_{t}^{(j)}\left(\ln \left(m_{x, t, i}\right)-a_{x, i}^{(j)}-B_{x}^{(j)} K_{t}^{(j)}-b_{x, i}^{(j)} k_{t, i}^{(j)}\right)
\end{aligned}
$$

and

$$
\left(\vec{r}_{b_{x, i}}^{(j)}\right)_{x-x_{\min }+1}=\sum_{t=t_{\min }}^{t_{\max }} k_{t, i}^{(j)}\left(\ln \left(m_{x, t, i}\right)-a_{x, i}^{(j)}-B_{x}^{(j)} K_{t}^{(j)}-b_{x, i}^{(j)} k_{t, i}^{(j)}\right),
$$

while $\vec{r}_{K}^{(j)}$ and $\vec{r}_{k_{i}}^{(j)}$ are column vectors with a dimension of $\left(t_{\max }-t_{\min }+1\right)$, whose $\left(t-t_{\min }+1\right)$ th elements are respectively given by

$$
\left(\vec{r}_{K}^{(j)}\right)_{t-t_{\min }+1}=\sum_{i=1}^{n_{p}} \sum_{x=x_{\min }}^{x_{\max }} B_{x}^{(j)}\left(\ln \left(m_{x, t, i}\right)-a_{x, i}^{(j)}-B_{x}^{(j)} K_{t}^{(j)}-b_{x, i}^{(j)} k_{t, i}^{(j)}\right)
$$

and

$$
\left(\vec{r}_{k_{i}}^{(j)}\right)_{t-t_{\min }+1}=\sum_{x=x_{\min }}^{x_{\max }} b_{x, i}^{(j)}\left(\ln \left(m_{x, t, i}\right)-a_{x, i}^{(j)}-B_{x}^{(j)} K_{t}^{(j)}-b_{x, i}^{(j)} k_{t, i}^{(j)}\right) .
$$

The smoothed ACF model is subject to an identifiability problem, which is typical among stochastic mortality models. For instance, if $\hat{B}_{x_{\min }}, \ldots, \hat{B}_{x_{\max }}$ and $\hat{K}_{t_{\min }}, \ldots, \hat{K}_{t_{\max }}$ are optimized parameter estimates, then $c \hat{B}_{x_{\min }}, \ldots, c \hat{B}_{x_{\max }}$ and $c^{-1} \hat{K}_{t_{\min }}, \ldots, c^{-1} \hat{K}_{t_{\max }}$, where $c$ is an arbitrary constant, are also optimized parameter estimates because they provide exactly the same fit. To stipulate parameter uniqueness, identifiability constraints are applied at the end of each iteration of the Newton's algorithm. Specifically, the parameter estimates in the $j$ th iteration are adjusted 
as follows:

$$
\left\{\begin{array}{l}
a_{x}^{(j)}=a_{x}^{(j)}+\frac{1}{t_{\max }-t_{\min }+1}\left(B_{x}^{(j)} \sum_{t=t_{\min }}^{t_{\max }} K_{t}^{(j)}+b_{x, i}^{(j)} \sum_{t=t_{\min }}^{t_{\max }} k_{x, i}^{(j)}\right) \\
K_{t}^{(j)}=\left(K_{t}^{(j)}-\frac{1}{t_{\max }-t_{\min }+1} \sum_{t=t_{\min }}^{t_{\max }} K_{t}^{(j)}\right) \sum_{x=x_{\min }}^{x_{\max }} B_{x}^{(j)} \\
k_{t, i}^{(j)}=\left(k_{t, i}^{(j)}-\frac{1}{t_{\max }-t_{\min }+1} \sum_{t=t_{\min }}^{t_{\max }} k_{x, i}^{(j)}\right) \sum_{x=x_{\min }}^{x_{\max }} b_{x, i}^{(j)} \\
B_{x}^{(j)}=B_{x}^{(j)} / \sum_{x=x_{\min }}^{x_{\max }} B_{x}^{(j)} \\
b_{x, i}^{(j)}=b_{x, i}^{(j)} / \sum_{x=x_{\min }}^{x_{\max }} b_{x, i}^{(j)}
\end{array}\right.
$$

In effect, the sums of the estimates of $B_{x}$ and $b_{x, i}$ over $x=x_{\min }, \ldots x_{\max }$ equal 1 , while the sums of the estimates of $K_{t}$ and $k_{t, i}$ over $t=t_{\min }, \ldots, t_{\max }$ equal 0 .

\subsection{Penalized Log-Likelihood for the ACF Model}

Another way to strike a balance between goodness-of-fit and smoothness is the method of penalized log-likelihood. We now generalize the penalized log-likelihood for the single-population Lee-Carter model to one for the multi-population ACF model.

Let us begin by stating the log-likelihood for the ACF model. Suppose that for population $i$, the random death count at age $x$ and year $t$ is $D_{x, t, i}$ and the corresponding non-random exposure count is $E_{x, t, i}$. To obtain a log-likelihood, a distributional assumption is needed. ${ }^{1}$ It is assumed that

$$
D_{x, t, i} \sim \operatorname{Poisson}\left(E_{x, t, i} \exp \left(a_{x, i}+B_{x} K_{t}+b_{x, i} k_{t, i}\right)\right) .
$$

Given this distributional assumption, the log-likelihood of the ACF model (excluding the constant terms) can be expressed as

$$
l=\sum_{i=1}^{n_{p}} \sum_{x=x_{\min }}^{x_{\max }} \sum_{t=t_{\min }}^{t_{\max }}\left(d_{x, t, i}\left(a_{x, i}+B_{x} K_{t}+b_{x, i} k_{t, i}\right)-E_{x, t, i} \exp \left(a_{x, i}+B_{x} K_{t}+b_{x, i} k_{t, i}\right)\right),
$$

where $d_{x, t, i}$ represents the realization of $D_{x, t, i}$. The penalized log-likelihood is then formulated by introducing a roughness penalty for each collection of age-response parameters:

$$
l-\frac{1}{2} \vec{B}^{\prime} \mathbf{P}_{c} \vec{B}-\frac{1}{2} \sum_{i=1}^{n_{p}} \vec{b}_{i}^{\prime} \mathbf{P}_{i} \vec{b}_{i}
$$

where the penalty matrices $\mathbf{P}_{c}$ and $\mathbf{P}_{i}$ are specified in equations (3.2) and (3.3), respectively. As in the method of penalized least squares, there are $\left(n_{p}+1\right)$ penalty parameters controlling the

\footnotetext{
${ }^{1}$ In contrast, the penalized least squares method does not require any distributional assumption.
} 
tradeoff between goodness-of-fit and smoothness. We recommend choosing the penalty parameters by the extended cross-validation that is detailed in Section 3.4.

It should be noted that although the log-likelihood specified by equation (3.6) is based on the assumption that the death counts at different values of $t, x$ and $i$ are conditionally independent, the death counts are dependent on one another when viewed unconditionally. The unconditional dependence structure is prescribed by the combination of parameters $a_{x, i}, B_{x}, K_{t}, b_{x, i}$ and $k_{t, i}$.

For a fixed set of smoothing parameters, the penalized log-likelihood estimates of the ACF model parameters can be obtained by maximizing the penalized log-likelihood. The Newton's algorithm for this optimization is the same as that specified in equation (3.4), but the diagonal matrices $\mathbf{C}_{a_{i}}^{(j)}, \mathbf{C}_{B}^{(j)}, \mathbf{C}_{b_{i}}^{(j)}, \mathbf{C}_{K}^{(j)}$ and $\mathbf{C}_{k_{i}}^{(j)}$ and the column vectors $\vec{r}_{a_{i}}^{(j)}, \vec{r}_{B}^{(j)}, \vec{r}_{b_{i}}^{(j)}, \vec{r}_{K}^{(j)}$ and $\vec{r}_{k_{i}}^{(j)}$ are specified differently. In particular, the $\left(x-x_{\min }+1, x-x_{\min }+1\right)$ th elements of $\mathbf{C}_{a_{i}}^{(j)}, \mathbf{C}_{B}^{(j)}$ and $\mathbf{C}_{b_{i}}^{(j)}$ are given by:

$$
\begin{aligned}
\left(\mathbf{C}_{a_{i}}^{(j)}\right)_{x-x_{\min }+1, x-x_{\min }+1} & =\sum_{t=t_{\min }}^{t_{\max }} E_{x, t, i} \exp \left(a_{x, i}^{(j)}+B_{x}^{(j)} K_{t}^{(j)}+b_{x, i}^{(j)} k_{t, i}^{(j)}\right) ; \\
\left(\mathbf{C}_{B}^{(j)}\right)_{x-x_{\min }+1, x-x_{\min }+1} & =\sum_{i=1}^{n_{p}} \sum_{t=t_{\min }}^{t_{\max }} E_{x, t, i}\left(K_{t}^{(j)}\right)^{2} \exp \left(a_{x, i}^{(j)}+B_{x}^{(j)} K_{t}^{(j)}+b_{x, i}^{(j)} k_{t, i}^{(j)}\right) ; \\
\left(\mathbf{C}_{b_{i}}^{(j)}\right)_{x-x_{\min }+1, x-x_{\min }+1} & =\sum_{t=t_{\min }}^{t_{\max }} E_{x, t, i}\left(k_{t, i}^{(j)}\right)^{2} \exp \left(a_{x, i}^{(j)}+B_{x}^{(j)} K_{t}^{(j)}+b_{x, i}^{(j)} k_{t, i}^{(j)}\right) ;
\end{aligned}
$$

the $\left(t-t_{\min }+1, t-t_{\min }+1\right)$ th elements in $\mathbf{C}_{K}^{(j)}$ and $\mathbf{C}_{k_{i}}^{(j)}$ are specified as:

$$
\begin{aligned}
\left(\mathbf{C}_{K}^{(j)}\right)_{t-t_{\min }+1, t-t_{\min }+1} & =\sum_{i=1}^{n_{p}} \sum_{x=x_{\min }}^{x_{\max }} E_{x, t, i}\left(B_{x}^{(j)}\right)^{2} \exp \left(a_{x, i}^{(j)}+B_{x}^{(j)} K_{t}^{(j)}+b_{x, i}^{(j)} k_{t, i}^{(j)}\right) ; \\
\left(\mathbf{C}_{k_{i}}^{(j)}\right)_{t-t_{\min }+1, t-t_{\min }+1} & =\sum_{x=x_{\min }}^{x_{\max }} E_{x, t, i}\left(b_{x, i}^{(j)}\right)^{2} \exp \left(a_{x, i}^{(j)}+B_{x}^{(j)} K_{t}^{(j)}+b_{x, i}^{(j)} k_{t, i}^{(j)}\right) ;
\end{aligned}
$$

the $\left(x-x_{\min }+1\right)$ th elements in $\vec{r}_{a_{i}}^{(j)}, \vec{r}_{B}^{(j)}$ and $\vec{r}_{b_{i}}^{(j)}$ are given by:

$$
\begin{aligned}
\left(\vec{r}_{a_{i}}^{(j)}\right)_{x-x_{\min }+1} & =\sum_{t=t_{\min }}^{t_{\max }}\left(d_{x, t, i}-E_{x, t, i} \exp \left(a_{x, i}^{(j)}+B_{x}^{(j)} K_{t}^{(j)}+b_{x, i}^{(j)} k_{t, i}^{(j)}\right)\right) \\
\left(\vec{r}_{B}^{(j)}\right)_{x-x_{\min }+1} & =\sum_{i=1}^{n_{p}} \sum_{t=t_{\min }}^{t_{\max }}\left(d_{x, t, i} K_{t}^{(j)}-E_{x, t, i} K_{t}^{(j)} \exp \left(a_{x, i}^{(j)}+B_{x}^{(j)} K_{t}^{(j)}+b_{x, i}^{(j)} k_{t, i}^{(j)}\right)\right) ; \\
\left(\vec{r}_{b_{i}}^{(j)}\right)_{x-x_{\min }+1} & =\sum_{t=t_{\min }}^{t_{\max }}\left(d_{x, t, i} k_{t, i}^{(j)}-E_{x, t, i} k_{t, i}^{(j)} \exp \left(a_{x, i}^{(j)}+B_{x}^{(j)} K_{t}^{(j)}+b_{x, i}^{(j)} k_{t, i}^{(j)}\right)\right)
\end{aligned}
$$


and finally the $\left(t-t_{\min }+1\right)$ th elements in $\vec{r}_{K}^{(j)}$ and $\vec{r}_{k_{i}}^{(j)}$ are specified as:

$$
\begin{aligned}
\left(\vec{r}_{K}^{(j)}\right)_{t-t_{\min }+1} & =\sum_{i=1}^{n_{p}} \sum_{x=x_{\min }}^{x_{\max }}\left(d_{x, t, i} B_{x}^{(j)}-E_{x, t, i} B_{x}^{(j)} \exp \left(a_{x, i}^{(j)}+B_{x}^{(j)} K_{t}^{(j)}+b_{x, i}^{(j)} k_{t, i}^{(j)}\right)\right) ; \\
\left(_{k_{i}}^{(j)}\right)_{t-t_{\min }+1} & =\sum_{x=x_{\min }}^{x_{\max }}\left(d_{x, t, i} b_{x, i}^{(j)}-E_{x, t, i} b_{x, i}^{(j)} \exp \left(a_{x, i}^{(j)}+B_{x}^{(j)} K_{t}^{(j)}+b_{x, i}^{(j)} k_{t, i}^{(j)}\right)\right) .
\end{aligned}
$$

As in the penalized least squares method, the identifiability constraints specified in equation (3.5) are applied at the end of each iteration in order to stipulate parameter uniqueness.

\subsection{Selecting the Smoothing Parameters with an Extended Cross-Validation}

We propose using an extended leave-one-out cross validation to determine the vector of smoothing parameters $\vec{\pi}=\left(\pi_{c}, \pi_{1}, \ldots, \pi_{n_{p}}\right)^{\prime}$. The extended cross-validation begins with an arbitrarily chosen $\vec{\pi}$. Given the chosen $\vec{\pi}$, we train (re-estimate) the ACF model by using the full data set except the observations at a certain age $x$ and in a certain year $t$. On the basis of the trained model, we calculate the sum of squared 'prediction errors' for the omitted observations.

For the method of penalized least squares, the sum of squared prediction errors is given by

$$
e_{x, t}^{2}(\vec{\pi})=\sum_{i=1}^{n_{p}}\left(\ln \left(m_{x, t, i}\right)-\hat{a}_{x, i, \vec{\pi}}^{-(x, t)}-\hat{B}_{x, \vec{\pi}}^{-(x, t)} \hat{K}_{t, \vec{\pi}}^{-(x, t)}-\hat{b}_{x, i, \vec{\pi}}^{-(x, t)} \hat{k}_{t, i, \vec{\pi}}^{-(x, t)}\right)^{2},
$$

where $\hat{a}_{x, i, \vec{\pi}}^{-(x, t)}, \hat{B}_{x, \vec{\pi}}^{-(x, t)}, \hat{K}_{t, \vec{\pi}}^{-(x, t)}, \hat{b}_{x, i, \vec{\pi}}^{-(x, t)}$ and $\hat{k}_{t, i, \vec{\pi}}^{-(x, t)}$ respectively represent the estimated values of $a_{x, i}, B_{x}, K_{t}, b_{x, i}$ and $k_{t, i}$ that are calculated from the training data set (in which the observations at age $x$ and in year $t$ are excluded).

For the method of penalized log-likelihood, the sum of squared prediction errors can be expressed as

$$
e_{x, t}^{2}(\vec{\pi})=\sum_{i=1}^{n_{p}}\left(\sqrt{2} \operatorname{sign}\left(d_{x, t, i}-\hat{d}_{x, t, i, \vec{\pi}}^{-(x, t)} \sqrt{d_{x, t, i} \ln \frac{d_{x, t, i}}{\hat{d}_{x, t, i, \vec{\pi}}^{-(x, t)}}-\left(d_{x, t, i}-\hat{d}_{x, t, i, \vec{\pi}}^{-(x, t)}\right)}\right)^{2},\right.
$$

where

$$
\hat{d}_{x, t, i, \vec{\pi}}^{-(x, t)}=E_{x, t, i} \exp \left(\hat{a}_{x, i, \vec{\pi}}^{-(x, t)}+\hat{B}_{x, \vec{\pi}}^{-(x, t)} \hat{K}_{t, \vec{\pi}}^{-(x, t)}+\hat{b}_{x, i, \vec{\pi}}^{-(x, t)} \hat{k}_{t, i, \vec{\pi}}^{-(x, t)}\right)
$$

represents the fitted number of death for age $x$, year $t$ and population $i$ on the basis of the parameter estimates calculated from the training data set.

By repeating the above for $x=x_{\min }, \ldots, x_{\max }$ and $t=t_{\min }, \ldots, t_{\max }$, we can obtain the aggregate sum of prediction errors:

$$
e_{\bullet, \bullet}^{2}(\vec{\pi})=\sum_{x=x_{\min }}^{x_{\max }} \sum_{t=t_{\min }}^{t_{\max }} e_{x, t}^{2}(\vec{\pi}) .
$$

The vector of smoothing parameter $\vec{\pi}$ is chosen so that $e_{\bullet \bullet \bullet}^{2}(\vec{\pi})$ is minimized. Following Delwarde et al. (2007), we find the optimal $\vec{\pi}$ by grid-searching over ranges of possible values. 

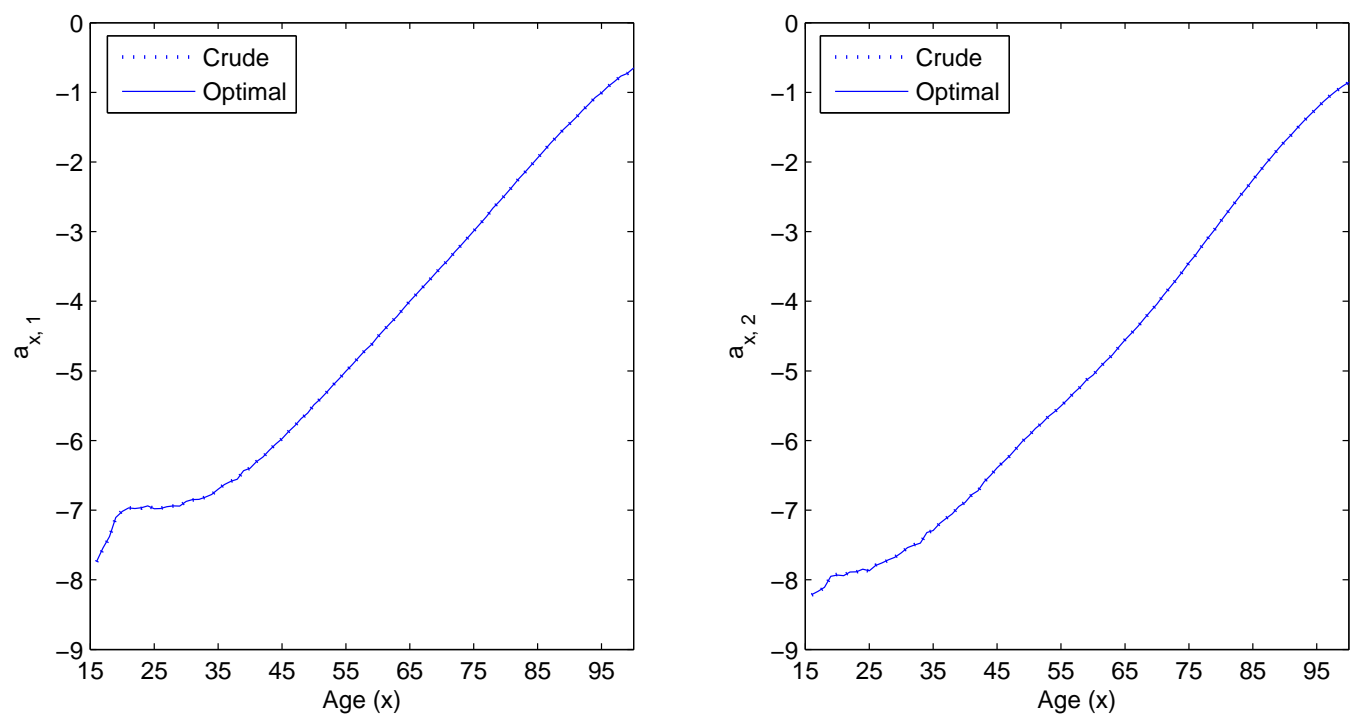

Figure 1: The maximum penalized $\log$-likelihood estimates of $a_{x, 1}$ (for Swedish males) and $a_{x, 2}$ (for Swedish females), crude $\left(\pi_{c}=\pi_{1}=\pi_{2}=0\right)$ and optimal $\left(\pi_{c}=10^{7}, \pi_{1}=10^{6}, \pi_{2}=10^{7}\right)$.

\section{An Application}

\subsection{Data}

We now provide an illustration using mortality data from the populations of Swedish males $(i=1)$ and Swedish females $(i=2)$. The data, which encompass raw death and exposure counts from $t_{\min }=1950$ to $t_{\max }=2011$ and from $x_{\min }=15$ to $x_{\max }=100$, are obtained from the Human Mortality Database. ${ }^{2}$

\subsection{Parameter Estimates}

In this illustration we focus on the method of penalized log-likelihood. On the basis of the extended cross-validation, it is found that the optimal smoothing parameters are $\pi_{c}=10^{7}, \pi_{1}=10^{6}$ and $\pi_{2}=10^{7}$.

Let us first focus on the estimates of $a_{x, 1}$ and $a_{x, 2}$, which are depicted in Figure 1. It can be observed that the crude maximum log-likelihood estimates (i.e., the estimates that are calculated at $\pi_{c}=\pi_{1}=\pi_{2}=0$ ) are already very smooth. This observation is in line with the empirical results produced by $\mathrm{Li}$ and Lee (2005), and justifies our decision of not imposing any roughness penalty on $a_{x, 1}$ and $a_{x, 2}$. The optimal maximum penalized log-likelihood estimates (i.e., the estimates that are calculated at the optimal values of $\pi_{c}, \pi_{1}$ and $\pi_{2}$ ) are very close to the crude ones. This result is expected, as no roughness penalty is imposed on $a_{x, 1}$ and $a_{x, 2}$.

\footnotetext{
${ }^{2}$ www.mortality.org
} 
Next, we study the crude and optimal estimates of $B_{x}, b_{x, 1}$ and $b_{x, 2}$, which are presented in the left panels of Figure 2. The crude estimates exhibit erratic patterns, especially over the age range of 15 to 45. The patterns of the optimal estimates across ages are much less erratic. To evaluate the effect of the smoothing parameters, in the right panels of Figure 2 we plot the estimates of $B_{x}, b_{x, 1}$ and $b_{x, 2}$ that are generated at various values of $\pi_{c}, \pi_{1}$, and $\pi_{2}$. It can be observed that a smoothing parameter that is higher than the optimal value tends to over-smooth the parameter series, while one that is lower than the optimal value gives the opposite result.

Finally, we examine the crude and optimal estimates of $K_{t}, k_{t, 1}$ and $k_{t, 2}$, which are displayed in Figure 3. Because no roughness penalty is imposed on $K_{t}, k_{t, 1}$ and $k_{t, 2}$, the crude and optimal estimates look similar. To obtain mortality forecasts, $K_{t}$ is further modeled by a random walk with drift, while $k_{t, 1}$ and $k_{t, 2}$ are further modeled by first order autoregressions. Given the way in which $K_{t}, k_{t, 1}$ and $k_{t, 2}$ are further modeled, the expected trajectories of $K_{t}, k_{t, 1}$ and $k_{t, 2}$ for $t>2011$ are smoothed straight lines or curves. Therefore, the progression of the projected mortality rates should be smooth over time, although the estimates of $K_{t}, k_{t, 1}$ and $k_{t, 2}$ over the calibration window are jagged.

\subsection{Mortality Forecasts}

To discern how the roughness penalties play a role in mortality forecasting, we compare the forecasts of log mortality rates that are derived from the crude and optimal parameter estimates. The results are shown in Figures 4 and 5 (for Swedish males) and Figures 6 and 7 (for Swedish females), with the left and right panels respectively representing the forecasts for $t=2021$ (10 years from the origin) and $t=2071$ (60 years from the origin). Without imposing any roughness penalty, the patterns of the forecasted log central death rates contain a substantial number of bumps that cannot be explained demographically. These bumps are either removed or dampened when the optimal roughness penalties are imposed. The effect of the roughness penalties is the most apparent over the age range of 15 to 45 , where the patterns of the crude estimates of $B_{x}$, $b_{x, 1}$ and $b_{x, 2}$ are the most erratic. It can also be seen that the effect of roughness penalties is stronger when the projection is made further into the future. This outcome arises because under the assumed process for $K_{t}$, the magnitude of the expected value of $K_{t}$ increases as $t$ increases. As a result, the problem associated with the estimates of $B_{x}$, which interact with the projected values of $K_{t}$, amplifies as we project further into the future.

\section{A Comparison with Alternative Approaches}

\subsection{Objectives of the Comparison}

In this section, we compare the proposed method with two alternative modeling approaches: the original ACF model (Li and Lee, 2005) that involves no parameter smoothing and the product- 

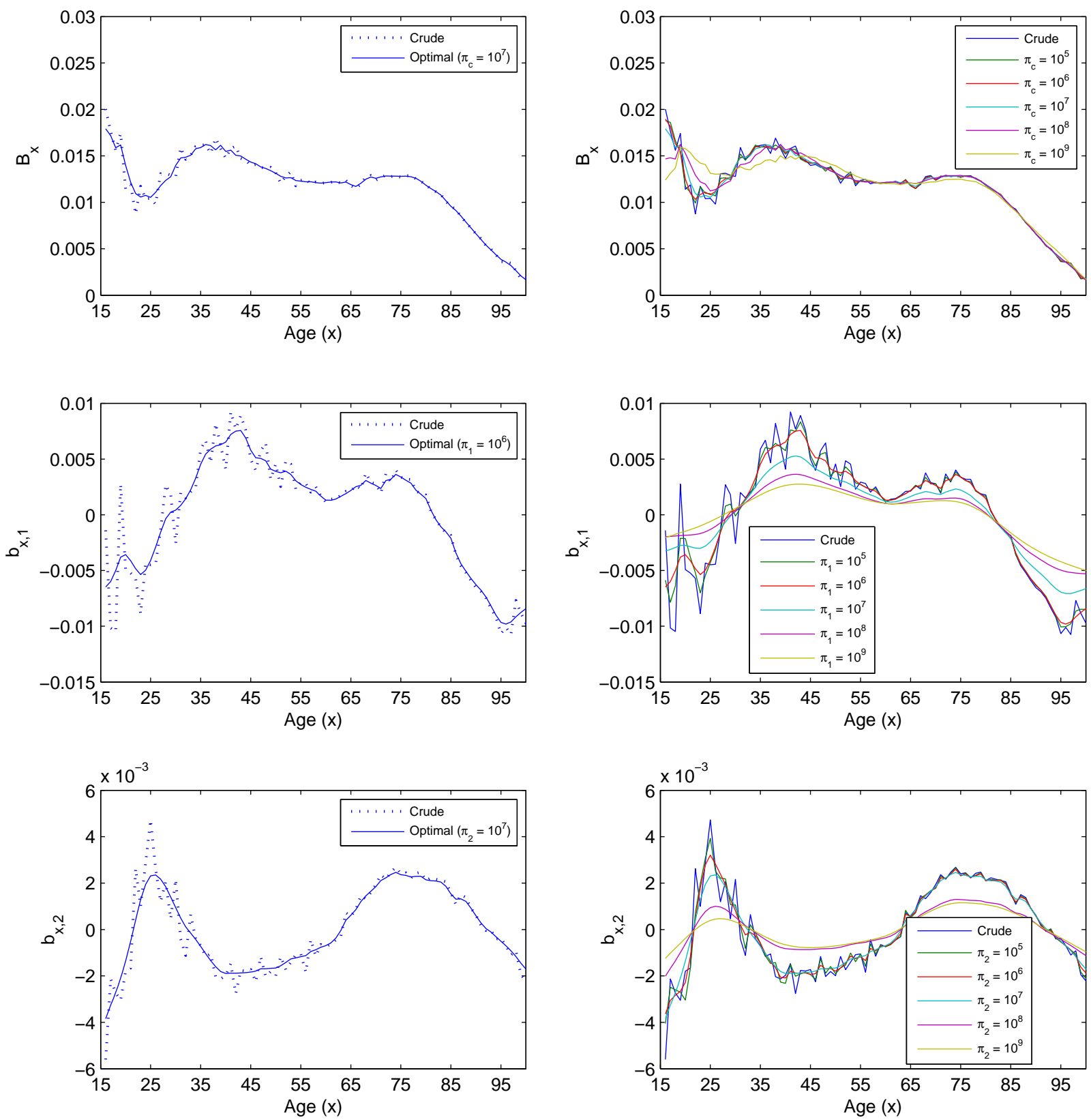

Figure 2: The maximum penalized log-likelihood estimates of $B_{x}$ (common to both populations), $b_{x, 1}$ (for Swedish males) and $b_{x, 2}$ (for Swedish females). The left panels compare the crude estimates $\left(\pi_{c}=\pi_{1}=\pi_{2}=0\right)$ against the optimal estimates $\left(\pi_{c}=10^{7}, \pi_{1}=10^{6}, \pi_{2}=10^{7}\right)$, whereas the right panels compare the estimates generated at various values of $\pi_{c}, \pi_{1}$, and $\pi_{2}$. 

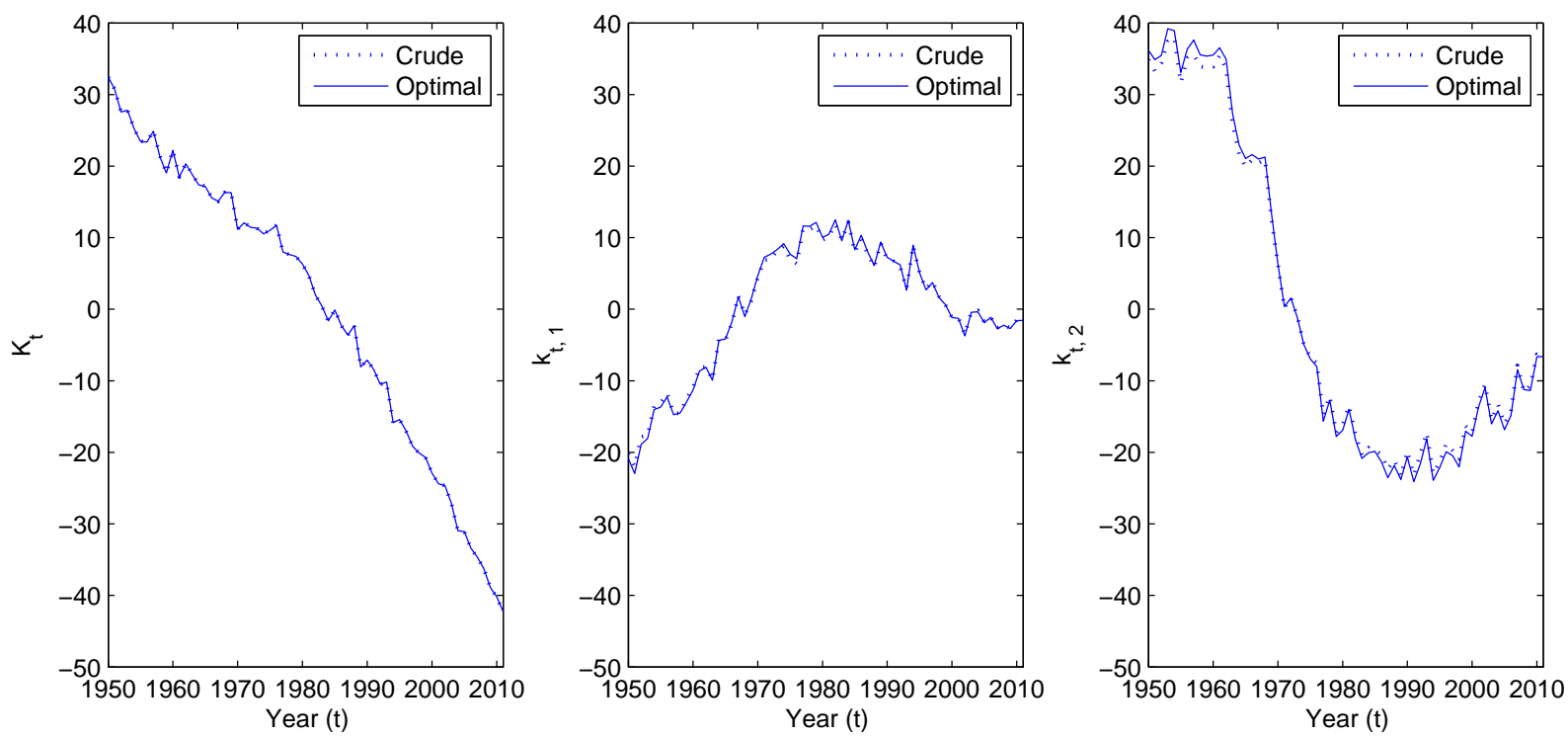

Figure 3: The maximum penalized log-likelihood estimates of $k_{t, 1}$ (for Swedish males) and $k_{t, 2}$ (for Swedish females), crude $\left(\pi_{c}=\pi_{1}=\pi_{2}=0\right)$ and optimal $\left(\pi_{c}=10^{7}, \pi_{1}=10^{6}, \pi_{2}=10^{7}\right)$.

ratio approach (Hyndman et al., 2013) in which smoothing is executed before the model parameters are estimated. The former is reviewed in Section 3.1, whereas the latter is explained in the next sub-section.

Our goal is not to choose the optimal approach out of the three candidates, as we believe that optimality depends heavily on the data under consideration and the forecaster's objectives. Instead, through this comparative study, we aim to gauge the reasonableness of the mortality forecasts produced by the proposed method (using the alternative modeling approaches as benchmarks) and to identify features that are distinctive to each of the modeling approaches.

For a more comprehensive comparison, we consider three different mortality data sets. The data sets used in this section are described in Table 1. Data Set I (identical to that used in Section 4) contains data from different genders of the same national population, Data Set II encompasses data from a national population and a sub-population of it, while Data Set III consists of data from different national populations which are geographically close. Except the data from the UK male insured lives (which are provided by the Institute and Faculty of Actuaries), all data used are obtained from the Human Mortality Database. For Data Set II, the age range ends with an open age group $(90+)$ because the data from the UK insured lives contains only a small number of death and exposure counts at ages 90 and beyond.

We consider three important aspects of mortality forecasting. First, we evaluate the models' 

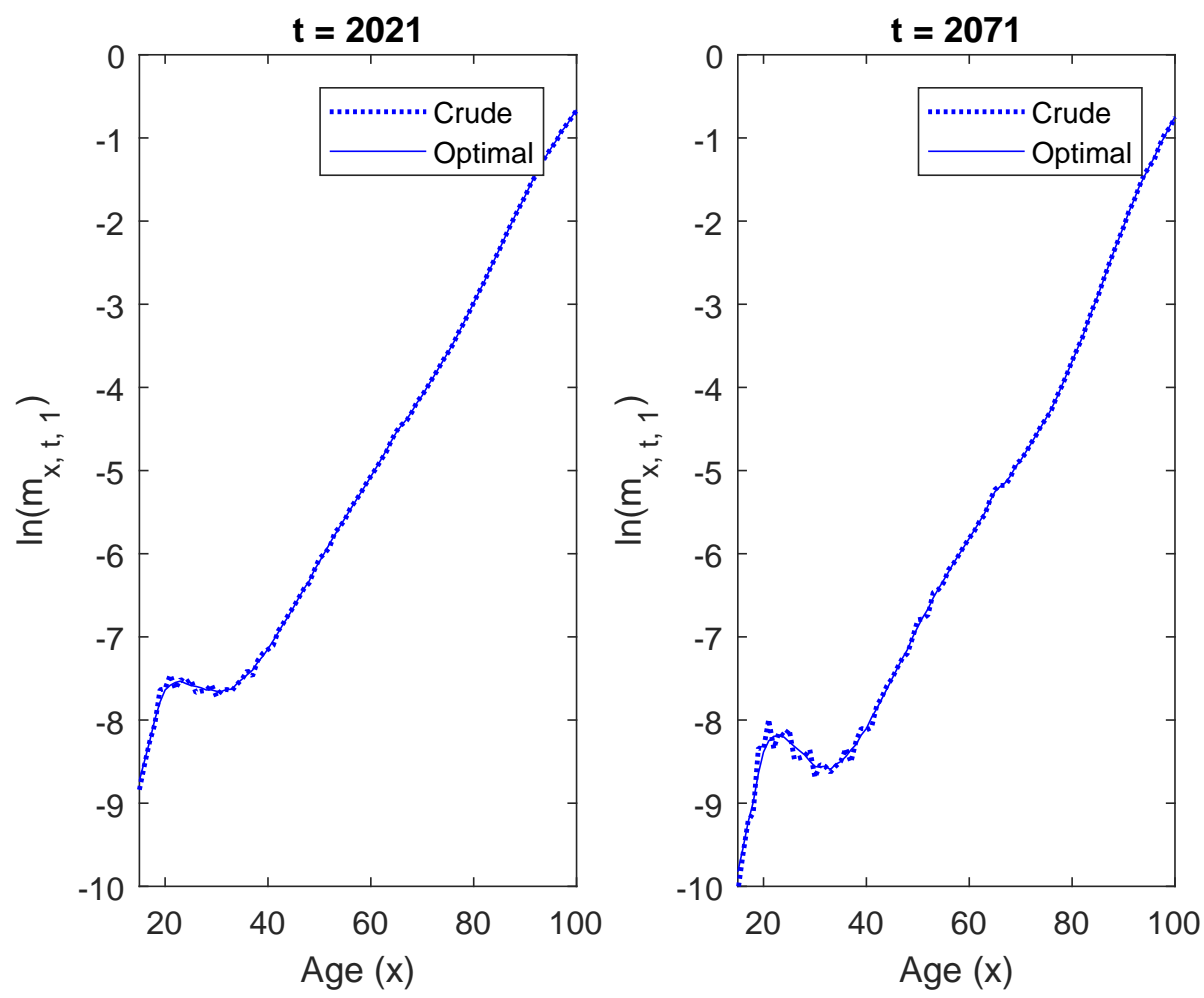

Figure 4: Forecasts of the $\log$ central death rates in years $t=2021$ (the left panels) and $t=2071$ (the right panels), on the basis of the crude and optimal parameter estimates, the whole age range of 15 to 100, population $i=1$ (Swedish males). 

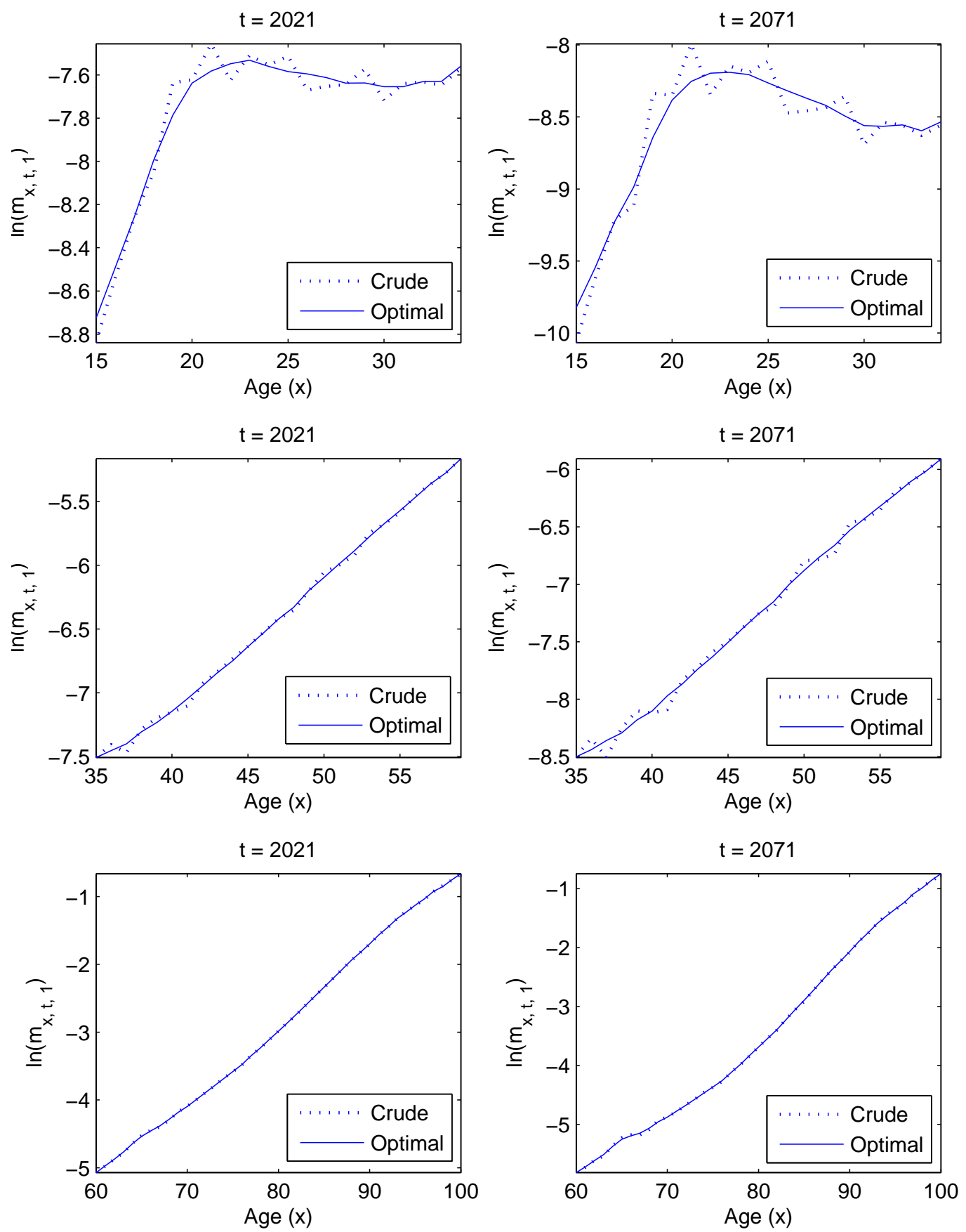

Figure 5: Forecasts of the $\log$ central death rates in years $t=2021$ (the left panels) and $t=2071$ (the right panels), on the basis of the crude and optimal parameter estimates, ages 0 to 34 (upper panels), ages 35 to 59 (middle panels) and ages 60 to 100 (lower panels), population $i=1$ (Swedish males). 

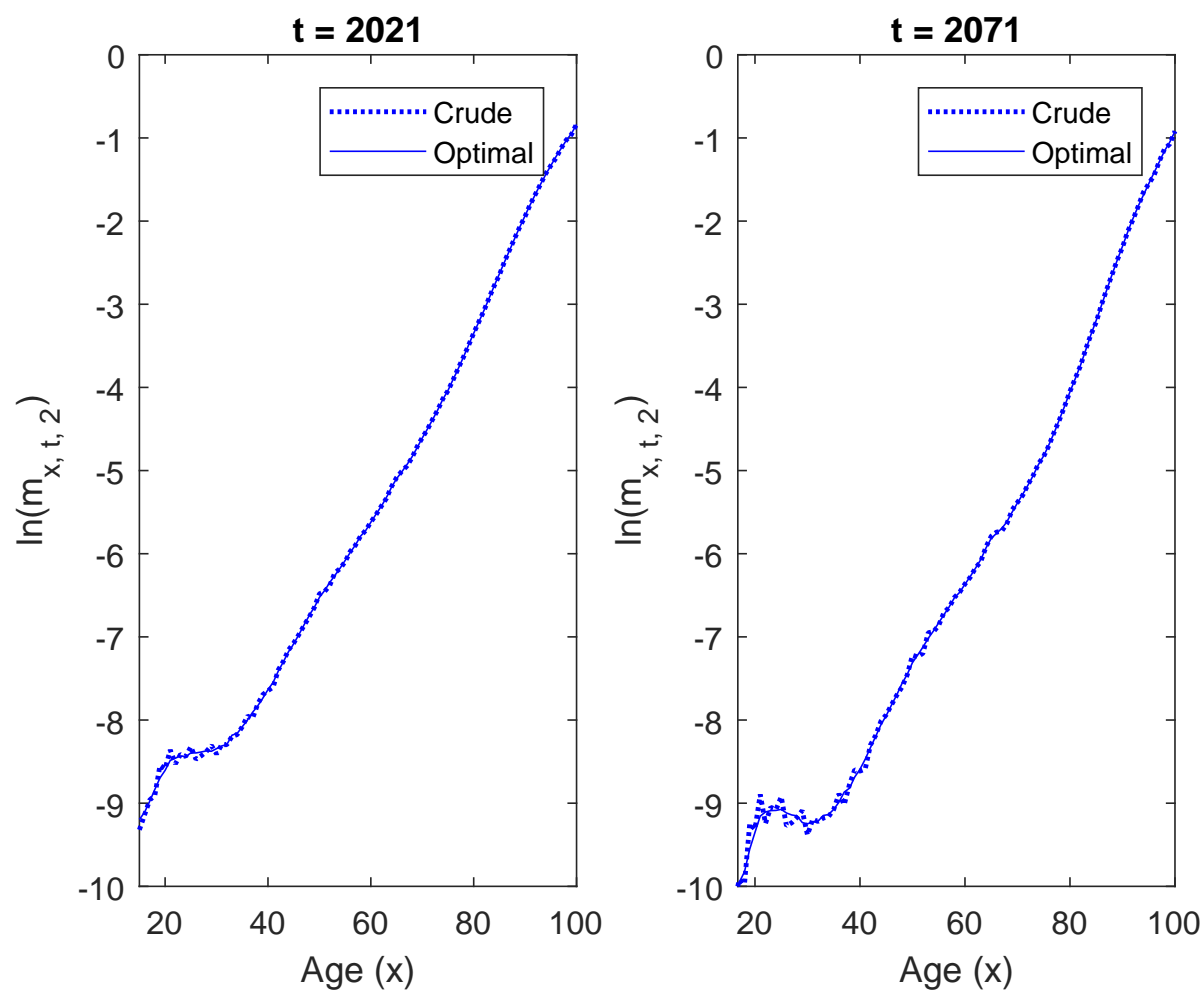

Figure 6: Forecasts of the $\log$ central death rates in years $t=2021$ (the left panels) and $t=2071$ (the right panels), on the basis of the crude and optimal parameter estimates, the whole age range of 15 to 100, population $i=2$ (Swedish females). 

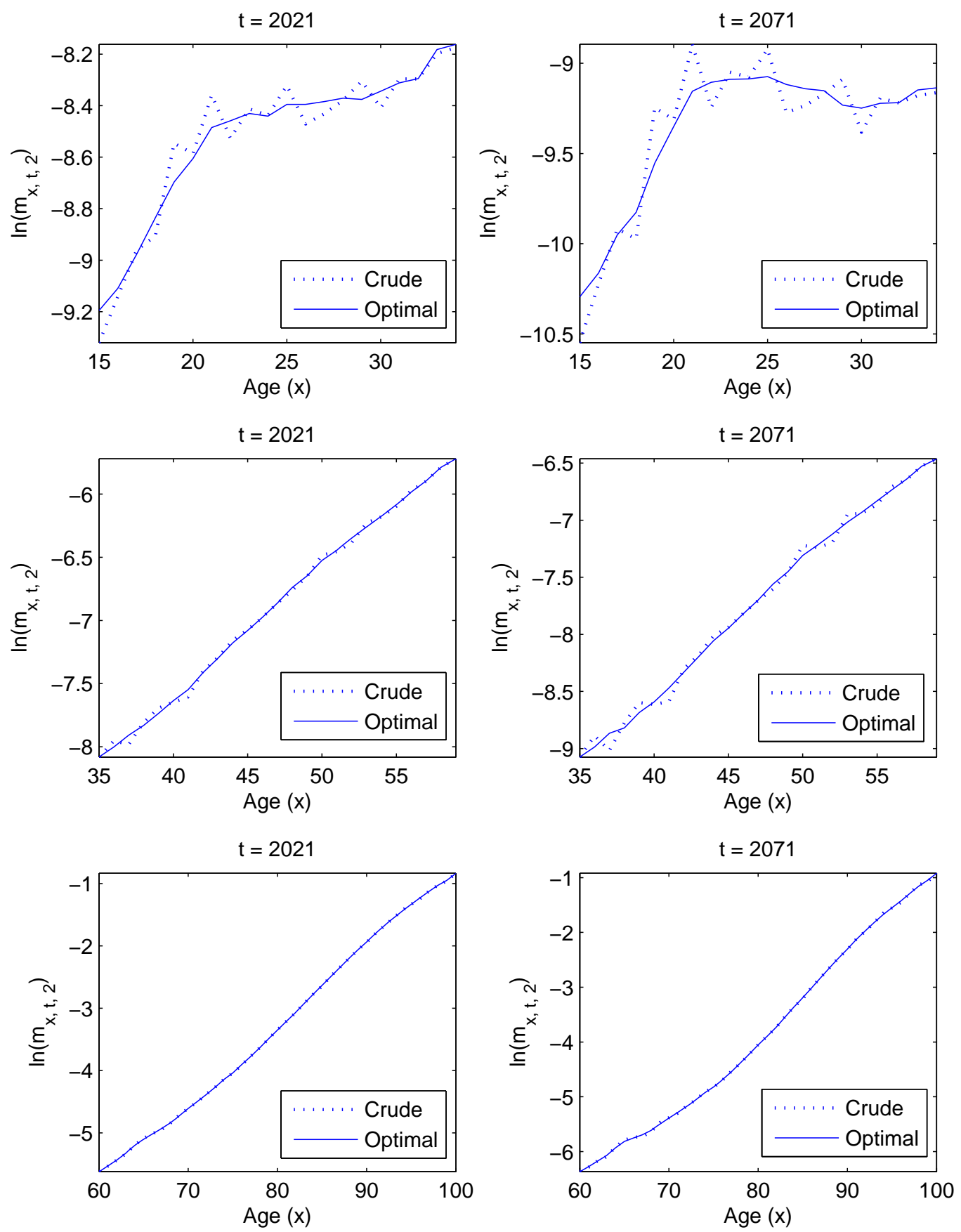

Figure 7: Forecasts of the $\log$ central death rates in years $t=2021$ (the left panels) and $t=2071$ (the right panels), on the basis of the crude and optimal parameter estimates, ages 0 to 34 (upper panels), ages 35 to 59 (middle panels) and ages 60 to 100 (lower panels), population $i=2$ (Swedish females). 
Table 1: The three data sets used in the comparative study.

\begin{tabular}{c|c|c|c|c}
\hline Data Set & Population 1 & Population 2 & Age range & Sample period \\
\hline I & Swedish males & Swedish females & 15 to 100 & 1950 to 2011 \\
II & English and Welsh males & UK male insured lives & 20 to $90+$ & 1947 to 2005 \\
III & US unisex & Canadian unisex & 0 to 100 & 1933 to 2011 \\
\hline
\end{tabular}

fitness to the historical data with the mean squared errors (MSE), which is defined as

$$
\operatorname{MSE}(i)=\sum_{x=x_{\min }}^{x_{\max }} \sum_{t=t_{\min }}^{t_{\max }}\left(\ln \left(m_{x, t, i}\right)-\ln \left(\hat{m}_{x, t, i}\right)\right)^{2}, \quad i=1,2,
$$

where $m_{x, t, i}$ and $\hat{m}_{x, t, i}$ respectively represent the observed (crude) and fitted central rates of death for population $i$, age $x$ and year $t$. A smaller MSE indicates a better goodness-of-fit.

Second, we evaluate the models' forecasting performance. To this end, we estimate the models to a restricted data sample, covering the first $s$ years in the original data sample (i.e., years $t_{\min }$ to $\left.t_{\min }+s-1\right)$. These models are then used to produce $h$-step-ahead 'forecasts' of age-specific central death rates (i.e., 'forecasts' of $m_{x, t_{\min }+s+h-1, i}$ for $x=x_{\min }, \ldots, x_{\max }$ and $i=1,2$ ). The procedure is repeated for $s=s_{\min }, \ldots, s_{\max }$, and the forecast accuracy is measured by the $h$-step root mean squared forecast errors (RMSFE), defined as

$$
\operatorname{RMSFE}(i, h)=\sqrt{\left(\frac{1}{n_{x} \times n_{s}} \sum_{x=x_{\min }}^{x_{\max }} \sum_{s=s_{\min }}^{s_{\max }}\left(\ln \left(m_{x, t_{\min }+s-1+h, i}\right)-\ln \left(\hat{m}_{x, t_{\min }+s-1+h, i \mid s}\right)\right)^{2}\right)},
$$

for $i=1,2$, where $n_{x}=x_{\max }-x_{\min }+1$ denotes the total number of ages encompassed by the data samples, $n_{s}=s_{\max }-s_{\min }+1$ represents the total number of restricted data samples under consideration, and $\hat{m}_{x, t_{\min }+s-1+h, i \mid s}$ is the $h$-step-ahead forecast of $m_{x, t_{\min }+s+h-1, i}$ generated from a model fitted to a restrictive data sample of size $s$ (covering years $t_{\min }$ to $t_{\min }+s-1$ ). We set $s_{\max }$ to $t_{\max }-t_{\min }-h+1$ (the maximum possible value given a forecast horizon of $h$ years), and $s_{\min }$ to 20 (for Data Sets I and II) and 30 (for Data Set III). ${ }^{3}$ A smaller RMSFE indicates a better forecasting performance.

Finally, we conduct a visual inspection of the age patterns of forecasted mortality at different future time points. In particular, we examine whether the stylized properties of age patterns of mortality are preserved in the forecasts.

\subsection{The Product-Ratio Approach}

The product-ratio approach can be regarded as a multi-population version of the Hyndman-Ullah approach (Hyndman and Ullah, 2007). Both approaches produce smoothed mortality forecasts

\footnotetext{
${ }^{3}$ We use a higher $s_{\min }$ for Data Set III on grounds that Data Set III is lengthier than the other two data sets. In this way, the denominators in equation (5.1) for all three data sets are of similar sizes.
} 
by smoothing historical mortality data prior to estimating model parameters. Both approaches can be implemented easily with the Demography Package in R (Hyndman et al., 2014). As each of the three data sets under consideration involves two populations, in the sequel we focus on the situation when $n_{p}=2$ populations are modeled unless otherwise specified.

\subsubsection{The Pre-Smoothing Procedure}

We begin by explaining the pre-smoothing procedure entailed in the product-ratio approach. The product-ratio approach assumes that there exists a sequence of smooth underlying central death rates, which are related to the corresponding observed (crude) central death rates in the following manner:

$$
\ln \left(m_{x, t, i}\right)=\ln \left(\tilde{m}_{x, t, i}\right)+\sigma_{x, i} \epsilon_{x, t, i}
$$

where $\tilde{m}_{x, t, i}$ represents the smoothed central death rate for age $x$, year $t$ and population $i, \epsilon_{x, t, i}$ is a standard normal random variable, and $\sigma_{x, i}$ is the observational variance which measures the

amount of noise surrounding $\ln \left(\tilde{m}_{x, t, i}\right)$ for each $x$ and $i$. It is further assumed that $\epsilon_{x_{1}, t_{1}, i_{1}}$ and $\epsilon_{x_{2}, t_{2}, i_{2}}$ are independent if $x_{1} \neq x_{2}, t_{1} \neq t_{2}$ or $i_{1} \neq i_{2}$.

The smooth underlying rates are estimated by fitting a weighted penalized regression spline (Wood, 1994) to $\left\{\ln \left(m_{x, t, i}\right) ; x=x_{\min }, \ldots, x_{\max }\right\}$ for each $t=t_{\min }, \ldots, t_{\max }$ and $i=1, \ldots, n_{p}$. Each regression spline is constrained so that the fitted central death rates are monotonically increasing for $x \geq 65$. Two parameters, $b$ and $k$, are needed when fitting the regression splines. Parameter $b$, where $0<b \leq 1$, represents the power transformation for age; $b=1$ corresponds to the situation in which no transformation is applied. Parameter $k$, where $k=1,2, \ldots$, represents the number of knots used. Given the chosen values of $b$ and $k$, the smoothing parameter $\lambda$ is optimized by a cross validation. In the Demography Package, the default values for $b$ and $k$ are 0.4 and 30, respectively. Having estimated the smooth underlying central death rates, the observational variance $\sigma_{x, i}$ for a given age $x$ and population $i$ can be estimated by using the sample variance of $\ln \left(m_{x, t, i}\right)-\ln \left(\tilde{m}_{x, t, i}\right)$ over $t=t_{\min }, \ldots, t_{\max }$.

\subsubsection{Modeling the Pre-Smoothed Mortality Rates}

The arrays of pre-smoothed central death rates are then transformed and modeled with functional time-series. The functional time-series used are as follows:

$$
\ln \left(p_{x, t}\right)=\mu_{x}^{p}+\sum_{j=1}^{N_{p}} B_{j, x} K_{j, t}+e_{x, t}^{p}
$$

and

$$
\ln \left(r_{x, t}\right)=\mu_{x}^{r}+\sum_{j=1}^{N_{r}} b_{j, x} k_{j, t}+e_{x, t}^{r}
$$


where $p_{x, t}=\sqrt{\tilde{m}_{x, t, 1} \times \tilde{m}_{x, t, 2}}, r_{x, t}=\sqrt{\tilde{m}_{x, t, 1} / \tilde{m}_{x, t, 2}}, N_{p}$ and $N_{r}$ are integers that are greater than or equal to $1, \mu_{x}^{p}, \mu_{x}^{r}, B_{j, x}, b_{j, x}$ are age-specific parameters, and $e_{x, t}^{p}$ and $e_{x, t}^{r}$ are the error terms that are assumed to have a zero mean and possess no serial correlations.

The estimation of the functional time-series involves multiple steps. First, parameters $\mu_{x}^{p}$ and $\mu_{x}^{p}$ are set to the averages of $\ln \left(p_{x, t}\right)$ and $\ln \left(r_{x, t}\right)$ over $t=t_{\min }, \ldots, t_{\max }$, respectively. Then, a principal component decomposition is applied to the matrix of $\ln \left(p_{x, t}\right)-\mu_{x}^{p}$, and $B_{j, x}$ and $K_{j, t}$ are set to the $j$ th principal component and principal component score, respectively. Likewise, another principal component decomposition is applied to the matrix of $\ln \left(r_{x, t}\right)-\mu_{x}^{r}$, and $b_{j, x}$ and $k_{j, t}$ are set to the $j$ th principal component and principal component score, respectively. When performing the estimation procedure, different weights may be applied to the values of $\ln \left(p_{x, t}\right)$ and $\ln \left(r_{x, t}\right)$ at different historical time points. In particular, more recent values may be weighted more heavily so that the resulting model lines up more closely with the most recent trends. However, as with estimating the other candidate models, the same weight is applied to all historical values when implementing the product-ratio approach. The issue of weighting historical observations is further discussed in Section 5.4.

To generate mortality forecasts, $K_{j, t}$ and $k_{j, t}$ are extrapolated with stochastic processes. For $K_{j, t}$, an appropriate $\operatorname{ARIMA}(p, d, q)$ process is used. ${ }^{4}$ For $k_{j, t}$, a mean-reverting process is needed in order to guarantee that the resulting forecasts are coherent. Possible choices include $\operatorname{ARIMA}(p, 0, q)$ and $\operatorname{ARFIMA}(p, d, q)$ processes. ${ }^{5}$

In the analysis, we set $N_{p}=1$ and $N_{r}=2$ so that the number of stochastic processes contained in the product-ratio model is the same as that in the ACF model and its smoothed version. This choice of $N_{p}$ and $N_{r}$ is somewhat justified, because for all three data sets under consideration more than $90 \%$ of variation in $\ln \left(p_{x, t}\right)$ is captured by the first principal component and more than $82 \%$ of the variation in $\ln \left(r_{x, t}\right)$ is captured by the two first principal components. Further, a $\operatorname{ARIMA}(0,1,0)$ process (i.e., a random walk with drift) is used for $K_{1, t}$ (the common trend) and $\operatorname{ARIMA}(1,0,0)$ processes (i.e., first-order autoregressions) are employed for $k_{1, t}$ and $k_{2, t}$ (the deviations from the common trend). This choice ensures a fair comparison, as the same processes are used in the ACF method to model the common trend and the deviations from it. We acknowledge that a better goodness-of-fit may be achieved by using more principal components (i.e., higher values of $N_{p}$ and $N_{r}$ ), and that a higher forecast accuracy may be obtained by using ARFIMA processes (rather than ARIMA processes) for $k_{1, t}$ and $k_{2, t}$. The choices of the processes for $k_{1, t}$ and $k_{2, t}$ are elaborated further in Section 5.4 .

\footnotetext{
${ }^{4}$ ARIMA stands for autoregressive integrated moving average. The triplet $(p, d, q)$, where $p, d, q$ are non-negative integers, is known as the order of the ARIMA process. For $K_{j, t}, d>0$ may be used as it may possess a stochastic trend.

${ }^{5}$ ARFIMA stands for autoregressive fractionally integrated moving average. In the triplet $(p, d, q), p$ and $q$ are non-negative integers and $-0.5<d<0.5$. By allowing $d$ to take on a fractional value, an ARFIMA process is well suited for a time-series that is stationary but exhibits too much long-range dependence to be modeled by an ARIMA process with $d=0$.
} 


\subsection{Empirical Results}

We now present the results of the comparison. To ease exposition, the following abbreviations are used throughout the rest of this section.

- ACF-O: The original ACF model (without any parameter smoothing)

- ACF-P: The ACF model that is estimated using the penalized log-likelihood method

- $\operatorname{PR}(b, k)$ : The product-ratio model that is based on pre-smoothing parameters $b$ and $k$

- PR: When the duplet $(b, k)$ is suppressed, the abbreviation refers to the product-ratio model that is based on the default values of $b$ and $k$ used in the Demography Package.

Table 2 shows the MSE values for all estimated models. For all populations under consideration, the ACF-P model yields a somewhat larger MSE value compared to the ACF-O model ${ }^{6}$, suggesting that the ACF-P model always under-performs when considering only goodness-of-fit. This observation highlights the tradeoff between goodness-of-fit and smoothness: to generate smoothed mortality forecasts with a penalized log-likelihood approach, a certain degree of goodness-of-fit must be sacrificed. Compared to the ACF-O and ACF-P models, the PR model (based on default values of $b$ and $k$ ) yields smaller MSE values for three populations but larger MSE values for the other three populations.

It is noteworthy that as we vary the pre-smoothing parameters $b$ (the power transformation for age) and $k$ (the number of knots), the MSE resulting from the $\operatorname{PR}(b, k)$ model varies considerably. The product-ratio method seems to be not so robust relative to (subjective) choices of $b$ and $k$. Although an end-user may adopt the default values of $b$ and $k$ prescribed by the Demography Package, it is unclear to the authors whether the default values are optimal.

Figure 8 displays the calculated values of $\operatorname{RMFSE}(i, h), h=1, \ldots, 30$, for all populations under consideration. The ACF-P model consistently produces more accurate forecasts compared to the ACF-O model. This outcome may be attributed to the fact that the ACF-O model carries the sampling errors entailed in parameter estimation forward to the projection period. The sampling errors accumulate as we project farther into the future, and therefore the gap between the RMFSE values resulting from the two models widens as the forecast horizon $h$ increases.

It is however unclear as to whether the product ratio method outperforms the ACF method in terms of forecast accuracy. For Data Set I, the PR model clearly outperforms the ACF-O and ACF-P models. For Data Set II, the PR model outperforms for longer term forecasts but not for shorter term ones. For Data Set III, the ACF-O and ACF-P model produces more accurate forecasts than the PR model for all forecast horizons considered. ${ }^{7}$

\footnotetext{
${ }^{6}$ For English and Welsh male and US unisex populations, the ACF-P model produces a larger MSE but the difference between the two MSE values is less than 0.0001 .

${ }^{7}$ One of the reviewers suggests that the under-performance of the PR model in Data Sets II and III might be attributed to the fact that these data sets are not taken from sub-populations of the same (larger) population.
} 


\begin{tabular}{|c|c|c|c|c|c|c|c|}
\hline \multicolumn{8}{|c|}{ Data Set I } \\
\hline \multicolumn{4}{|c|}{ Swedish Male } & \multicolumn{4}{|c|}{ Swedish Female } \\
\hline ACF-O & \multicolumn{3}{|c|}{0.0134} & $\mathrm{ACF}-\mathrm{O}$ & \multicolumn{3}{|c|}{0.0222} \\
\hline ACF-P & \multicolumn{3}{|c|}{0.0137} & ACF-P & \multicolumn{3}{|c|}{0.0225} \\
\hline $\mathrm{PR}$ & \multicolumn{3}{|c|}{0.0138} & PR & \multicolumn{3}{|c|}{0.0197} \\
\hline $\operatorname{PR}(0.1,30)$ & 0.0136 & $\operatorname{PR}(0.4,1)$ & 0.0226 & $\operatorname{PR}(0.1,30)$ & 0.0195 & $\operatorname{PR}(0.4,1)$ & 0.0344 \\
\hline $\operatorname{PR}(0.2,30)$ & 0.0136 & $\operatorname{PR}(0.4,4)$ & 0.0226 & $\operatorname{PR}(0.2,30)$ & 0.0196 & $\operatorname{PR}(0.4,4)$ & 0.0344 \\
\hline $\operatorname{PR}(0.3,30)$ & 0.0137 & $\operatorname{PR}(0.4,7)$ & 0.0159 & $\operatorname{PR}(0.3,30)$ & 0.0197 & $\operatorname{PR}(0.4,7)$ & 0.0207 \\
\hline $\operatorname{PR}(0.4,30)$ & 0.0138 & $\operatorname{PR}(0.4,10)$ & 0.0140 & $\operatorname{PR}(0.4,30)$ & 0.0197 & $\operatorname{PR}(0.4,10)$ & 0.0200 \\
\hline $\operatorname{PR}(0.5,30)$ & 0.0140 & $\operatorname{PR}(0.4,13)$ & 0.0134 & $\operatorname{PR}(0.5,30)$ & 0.0197 & $\operatorname{PR}(0.4,13)$ & 0.0197 \\
\hline $\operatorname{PR}(0.6,30)$ & 0.0142 & $\operatorname{PR}(0.4,16)$ & 0.0136 & $\operatorname{PR}(0.6,30)$ & 0.0199 & $\operatorname{PR}(0.4,16)$ & 0.0198 \\
\hline $\operatorname{PR}(0.7,30)$ & 0.0144 & $\operatorname{PR}(0.4,19)$ & 0.0137 & $\operatorname{PR}(0.7,30)$ & 0.0200 & $\operatorname{PR}(0.4,19)$ & 0.0197 \\
\hline $\operatorname{PR}(0.8,30)$ & 0.0146 & $\operatorname{PR}(0.4,22)$ & 0.0138 & $\operatorname{PR}(0.8,30)$ & 0.0200 & $\operatorname{PR}(0.4,22)$ & 0.0197 \\
\hline $\operatorname{PR}(0.9,30)$ & 0.0149 & $\operatorname{PR}(0.4,25)$ & 0.0139 & $\operatorname{PR}(0.9,30)$ & 0.0201 & $\operatorname{PR}(0.4,25)$ & 0.0198 \\
\hline $\operatorname{PR}(1.0,30)$ & 0.0151 & $\operatorname{PR}(0.4,28)$ & 0.0138 & $\operatorname{PR}(1.0,30)$ & 0.0202 & $\operatorname{PR}(0.4,28)$ & 0.0197 \\
\hline \multicolumn{8}{|c|}{ Data Set II } \\
\hline \multicolumn{4}{|c|}{ English and Welsh Male } & \multicolumn{4}{|c|}{ UK Male Insured Lives } \\
\hline $\mathrm{ACF}-\mathrm{O}$ & \multicolumn{3}{|c|}{0.0026} & $\mathrm{ACF}-\mathrm{O}$ & \multicolumn{3}{|c|}{0.0343} \\
\hline ACF-P & \multicolumn{3}{|c|}{0.0026} & ACF-P & \multicolumn{3}{|c|}{0.0348} \\
\hline $\mathrm{PR}$ & \multicolumn{3}{|c|}{0.0087} & $\mathrm{PR}$ & \multicolumn{3}{|c|}{0.0302} \\
\hline $\operatorname{PR}(0.1,30)$ & 0.0087 & $\operatorname{PR}(0.4,1)$ & 0.0134 & $\operatorname{PR}(0.1,30)$ & 0.0300 & $\operatorname{PR}(0.4,1)$ & 0.0360 \\
\hline $\operatorname{PR}(0.2,30)$ & 0.0087 & $\operatorname{PR}(0.4,4)$ & 0.0134 & $\operatorname{PR}(0.2,30)$ & 0.0300 & $\operatorname{PR}(0.4,4)$ & 0.0360 \\
\hline $\operatorname{PR}(0.3,30)$ & 0.0087 & $\operatorname{PR}(0.4,7)$ & 0.0091 & $\operatorname{PR}(0.3,30)$ & 0.0301 & $\operatorname{PR}(0.4,7)$ & 0.0302 \\
\hline $\operatorname{PR}(0.4,30)$ & 0.0087 & $\operatorname{PR}(0.4,10)$ & 0.0090 & $\operatorname{PR}(0.4,30)$ & 0.0302 & $\operatorname{PR}(0.4,10)$ & 0.0301 \\
\hline $\operatorname{PR}(0.5,30)$ & 0.0087 & $\operatorname{PR}(0.4,13)$ & 0.0089 & $\operatorname{PR}(0.5,30)$ & 0.0303 & $\operatorname{PR}(0.4,13)$ & 0.0303 \\
\hline $\operatorname{PR}(0.6,30)$ & 0.0086 & $\operatorname{PR}(0.4,16)$ & 0.0087 & $\operatorname{PR}(0.6,30)$ & 0.0304 & $\operatorname{PR}(0.4,16)$ & 0.0304 \\
\hline $\operatorname{PR}(0.7,30)$ & 0.0086 & $\operatorname{PR}(0.4,19)$ & 0.0087 & $\operatorname{PR}(0.7,30)$ & 0.0306 & $\operatorname{PR}(0.4,19)$ & 0.0304 \\
\hline $\operatorname{PR}(0.8,30)$ & 0.0085 & $\operatorname{PR}(0.4,22)$ & 0.0086 & $\operatorname{PR}(0.8,30)$ & 0.0307 & $\operatorname{PR}(0.4,22)$ & 0.0303 \\
\hline $\operatorname{PR}(0.9,30)$ & 0.0085 & $\operatorname{PR}(0.4,25)$ & 0.0087 & $\operatorname{PR}(0.9,30)$ & 0.0309 & $\operatorname{PR}(0.4,25)$ & 0.0302 \\
\hline $\operatorname{PR}(1.0,30)$ & 0.0085 & $\operatorname{PR}(0.4,28)$ & 0.0087 & $\operatorname{PR}(1.0,30)$ & 0.0310 & $\operatorname{PR}(0.4,28)$ & 0.0302 \\
\hline \multicolumn{8}{|c|}{ Data Set III } \\
\hline \multicolumn{4}{|c|}{ US Unisex } & \multicolumn{4}{|c|}{ Canadian Unisex } \\
\hline $\mathrm{ACF}-\mathrm{O}$ & \multicolumn{3}{|c|}{0.0024} & $\mathrm{ACF}-\mathrm{O}$ & \multicolumn{3}{|c|}{0.0104} \\
\hline ACF-P & & 0.0024 & & ACF-P & & 0.0107 & \\
\hline $\mathrm{PR}$ & & 0.0060 & & $\mathrm{PR}$ & & 0.0089 & \\
\hline $\operatorname{PR}(0.1,30)$ & 0.0060 & $\operatorname{PR}(0.4,1)$ & 0.1580 & $\operatorname{PR}(0.1,30)$ & 0.0089 & $\operatorname{PR}(0.4,1)$ & 0.1350 \\
\hline $\operatorname{PR}(0.2,30)$ & 0.0060 & $\operatorname{PR}(0.4,4)$ & 0.1580 & $\operatorname{PR}(0.2,30)$ & 0.0089 & $\operatorname{PR}(0.4,4)$ & 0.1350 \\
\hline $\operatorname{PR}(0.3,30)$ & 0.0060 & $\operatorname{PR}(0.4,7)$ & 0.0351 & $\operatorname{PR}(0.3,30)$ & 0.0089 & $\operatorname{PR}(0.4,7)$ & 0.0352 \\
\hline $\operatorname{PR}(0.4,30)$ & 0.0060 & $\operatorname{PR}(0.4,10)$ & 0.0121 & $\operatorname{PR}(0.4,30)$ & 0.0089 & $\operatorname{PR}(0.4,10)$ & 0.0149 \\
\hline $\operatorname{PR}(0.5,30)$ & 0.0060 & $\operatorname{PR}(0.4,13)$ & 0.0072 & $\operatorname{PR}(0.5,30)$ & 0.0088 & $\operatorname{PR}(0.4,13)$ & 0.0100 \\
\hline $\operatorname{PR}(0.6,30)$ & 0.0059 & $\operatorname{PR}(0.4,16)$ & 0.0065 & $\operatorname{PR}(0.6,30)$ & 0.0087 & $\operatorname{PR}(0.4,16)$ & 0.0091 \\
\hline $\operatorname{PR}(0.7,30)$ & 0.0061 & $\operatorname{PR}(0.4,19)$ & 0.0060 & $\operatorname{PR}(0.7,30)$ & 0.0088 & $\operatorname{PR}(0.4,19)$ & 0.0089 \\
\hline $\operatorname{PR}(0.8,30)$ & 0.0073 & $\operatorname{PR}(0.4,22)$ & 0.0060 & $\operatorname{PR}(0.8,30)$ & 0.0098 & $\operatorname{PR}(0.4,22)$ & 0.0088 \\
\hline $\operatorname{PR}(0.9,30)$ & 0.0126 & $\operatorname{PR}(0.4,25)$ & 0.0060 & $\operatorname{PR}(0.9,30)$ & 0.0151 & $\operatorname{PR}(0.4,25)$ & 0.0088 \\
\hline $\operatorname{PR}(1.0,30)$ & 0.0239 & $\operatorname{PR}(0.4,28)$ & 0.0060 & $\operatorname{PR}(1.0,30)$ & 0.0270 & $\operatorname{PR}(0.4,28)$ & 0.0088 \\
\hline
\end{tabular}

Table 2: The MSE values for all estimated models. 
Data Set I: Swedish Males

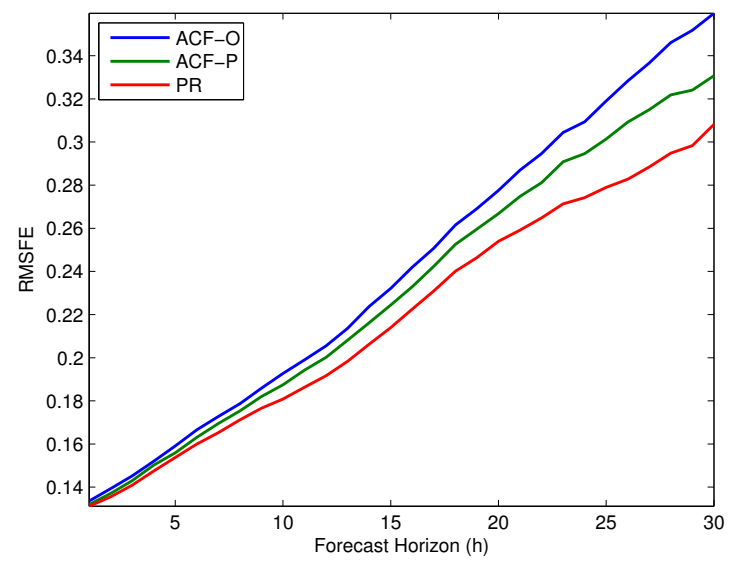

Data Set II: English and Welsh Males

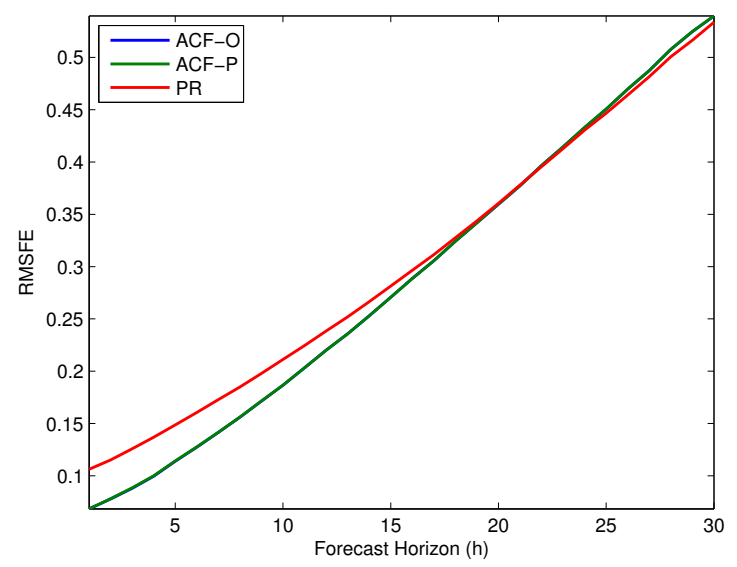

Data Set III: US Unisex

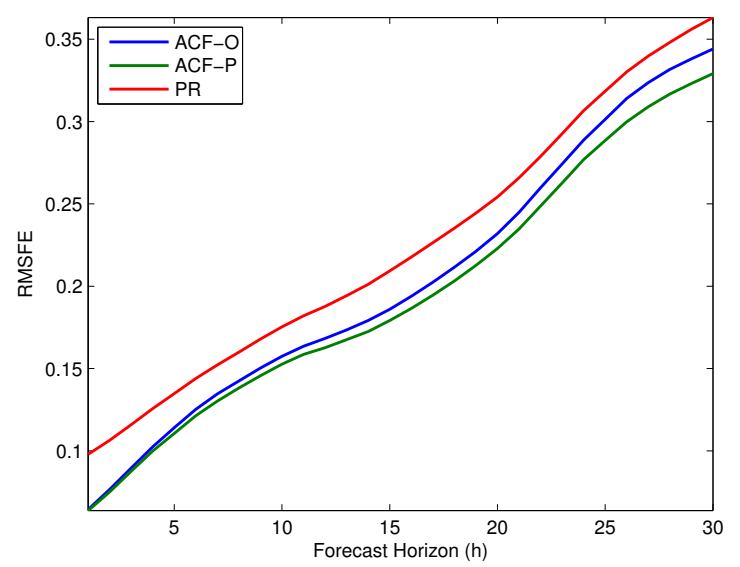

Data Set I: Swedish Females

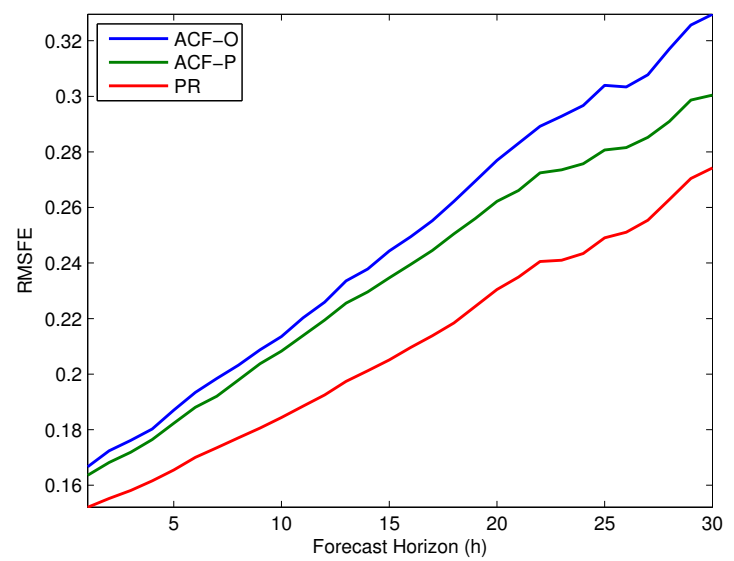

Data Set II: UK Male Insured Lives

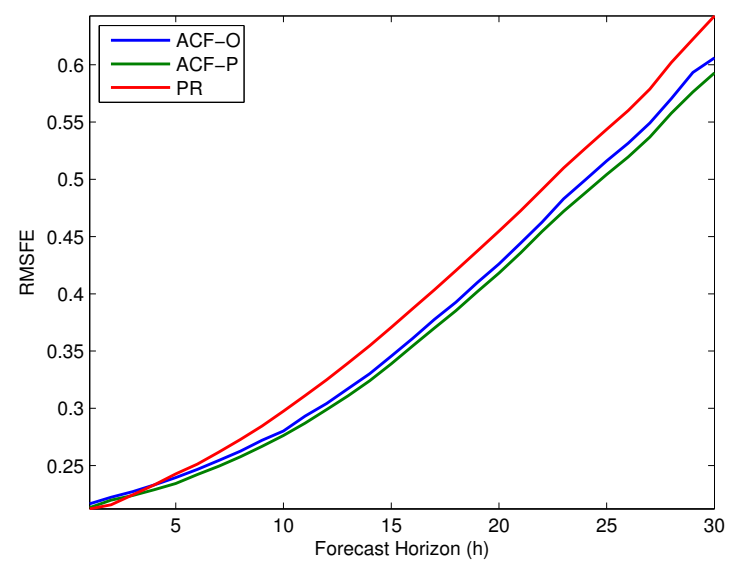

Data Set III: Canadian Unisex

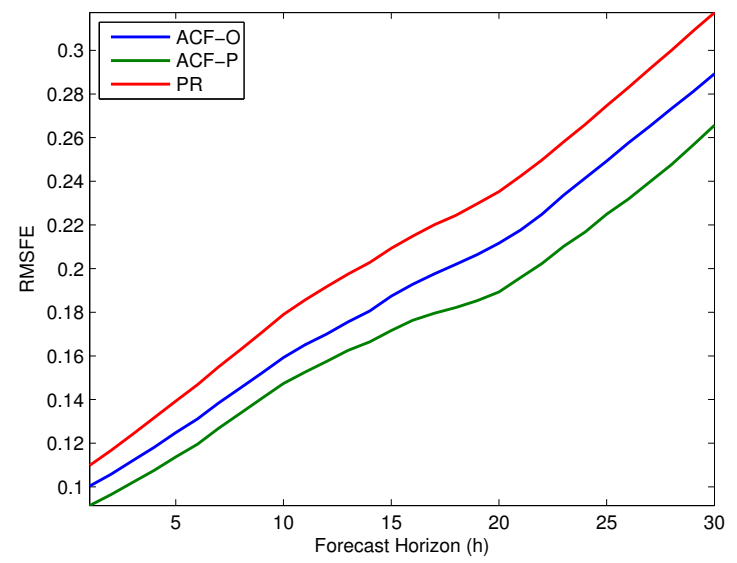

Figure 8: The values of RMFSE produced by the ACF-O, ACF-P and PR models when they are fitted to the three data sets under consideration. 
Finally, we evaluate the projected age pattern of mortality rates (Figures 9 and 10). For the sake of space, we only display the results generated from Data Set I. As expected, the forecasted age patterns of mortality rates produced by the ACF-P and PR models are smooth, while those produced from the ACF-O model are not. All forecasted age patterns appear to be reasonable. However, for females, the pattern of the mortality rates projected using the PR model from age 15 to 30 appears to be too flat relative to the expected 'accident hump' that is captured by the ACF-O and ACF-P models. This observation suggests that the product-ratio approach may be subject to an over-smoothing problem.

\subsection{Discussion}

To recap, for a fair comparison the results presented in the previous sub-section are based on the following setting:

- When estimating all three candidate models, historical observations are equally weighted.

- We set $N_{p}=1$ and $N_{r}=2$, so that all three candidate models contain the same number of indexes for the common trend as well as the same number of indexes for the deviations from the common trend.

- The same type time-series processes are used in all three candidate models: a random walk with drift for the common trend and first-order autoregressive processes for the deviations from the common trend.

The results presented in the previous sub-section therefore reflect how fitting and forecasting performances may be different when (1) smoothing is applied in different stages (prior to estimation or within the estimation process) and (2) the model structure is different. We now explain the structural similarities and differences between the ACF and PR models under the setting summarized above.

When modeling $n_{p}=2$ populations, the ACF model (with or without parameter smoothing) can be expressed as

$$
\left\{\begin{array}{l}
\ln \left(m_{x, t, 1}\right)=a_{x, 1}+B_{x} K_{t}+b_{x, 1} k_{t, 1}+\varepsilon_{x, t, 1} \\
\ln \left(m_{x, t, 2}\right)=a_{x, 2}+B_{x} K_{t}+b_{x, 2} k_{t, 2}+\varepsilon_{x, t, 2}
\end{array},\right.
$$

and the PR model (with $N_{p}=1$ and $N_{r}=2$ ) can be rewritten as

$$
\left\{\begin{array}{l}
\ln \left(\tilde{m}_{x, t, 1}\right)=\left(\mu_{x}^{p}+\mu_{x}^{r}\right)+B_{x} K_{t}+b_{x, 1} k_{t, 1}+b_{x, 2} k_{t, 2}+e_{x, t}^{p}+e_{x, t}^{r} \\
\ln \left(\tilde{m}_{x, t, 2}\right)=\left(\mu_{x}^{p}-\mu_{x}^{r}\right)+B_{x} K_{t}-b_{x, 1} k_{t, 1}-b_{x, 2} k_{t, 2}+e_{x, t}^{p}-e_{x, t}^{r}
\end{array} .\right.
$$

We can then easily observe the similarities between the two model structures. When fitted to the same data set, the two model structures contain the same number of parameters and the same number of error terms. Also, in both models, the common trend is captured by $K_{t}$ (further 
Swedish male; 1-year forecast horizon
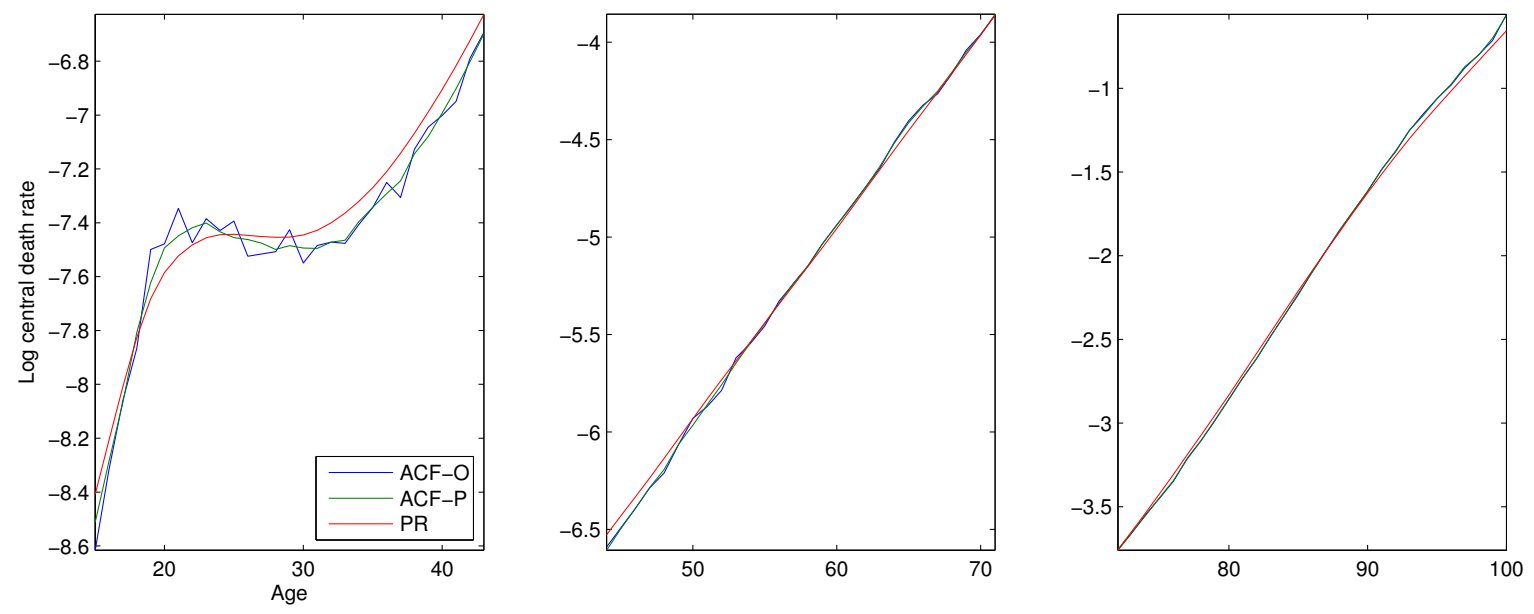

Swedish male; 10-year forecast horizon
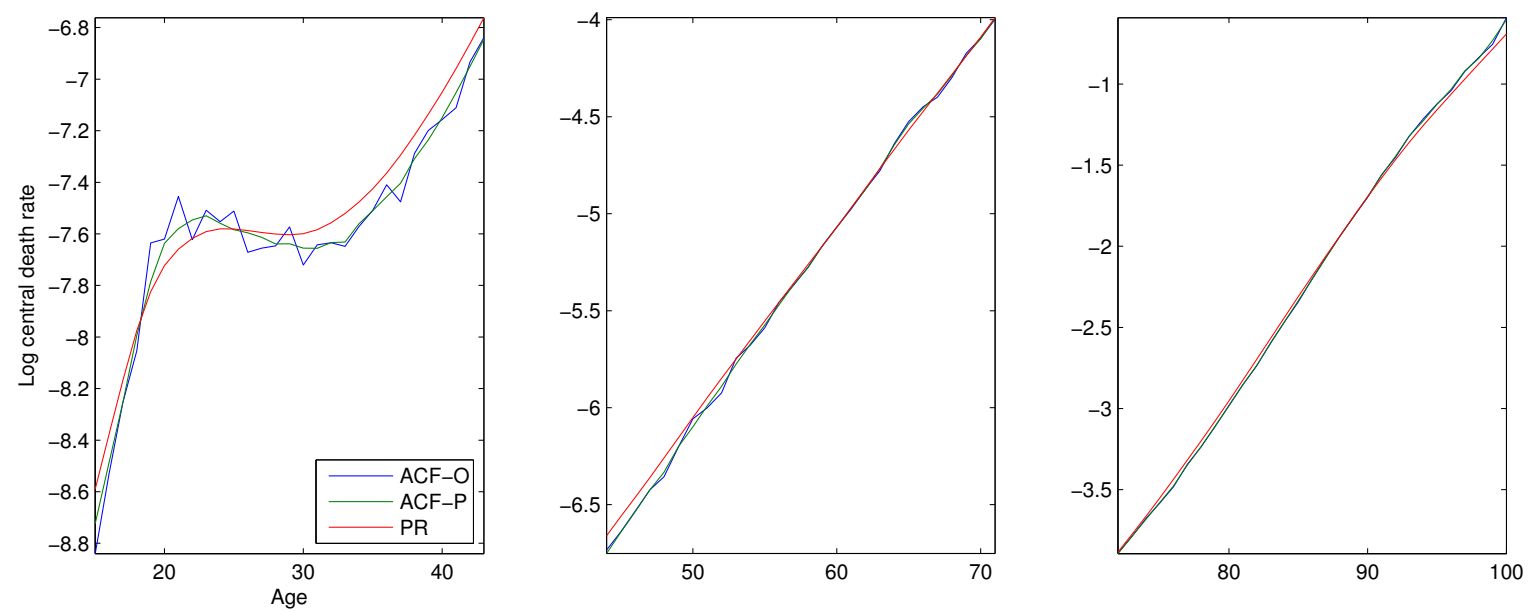

Swedish male; 60-year forecast horizon
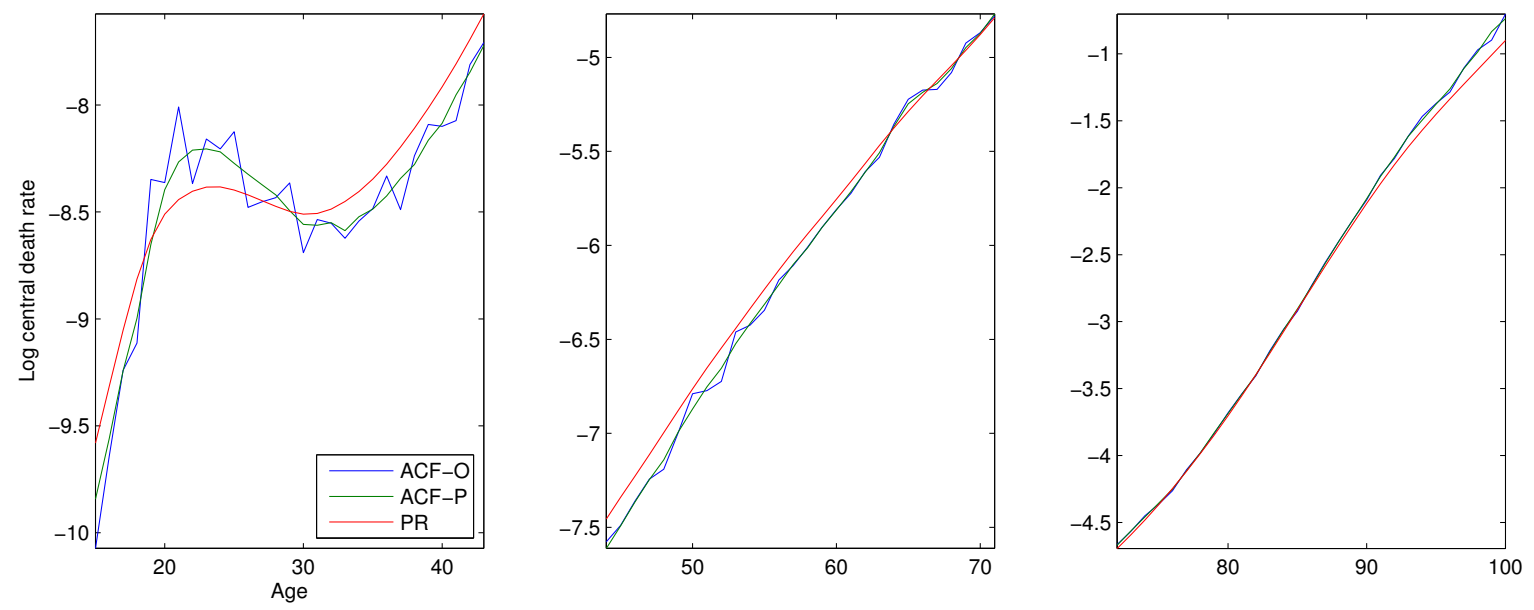

Figure 9: Forecasts of the log central death rates for Swedish male, 1, 10 and 60 year(s) beyond the forecast origin, generated from the ACF-O, ACF-P and PR models. 
Swedish females; 1-year forecast horizon
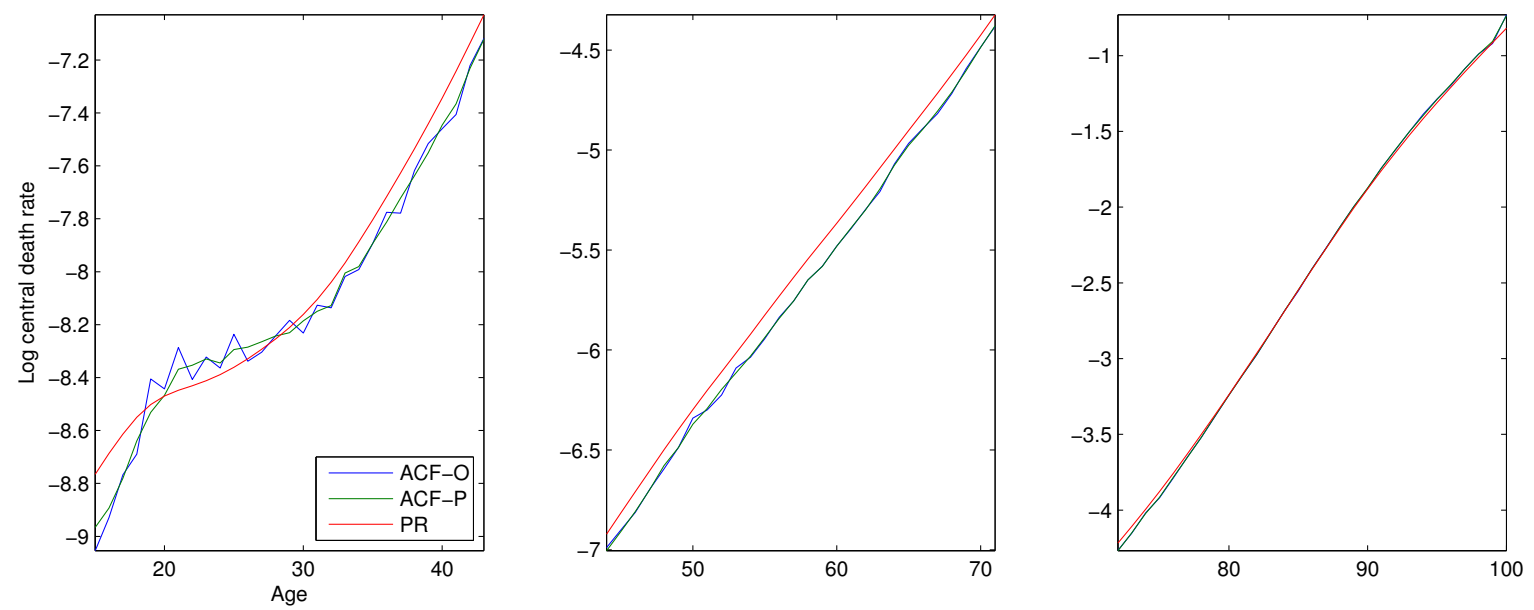

Swedish females; 10-year forecast horizon
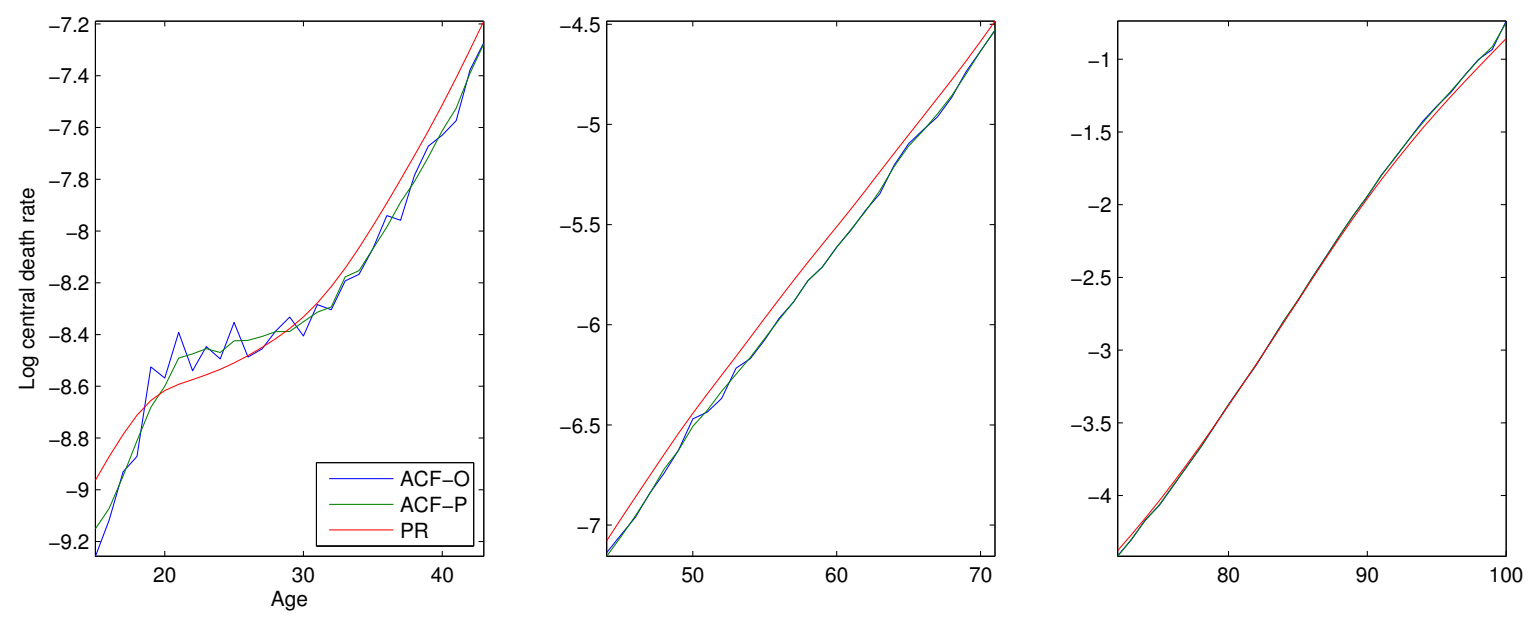

Swedish females; 60-year forecast horizon
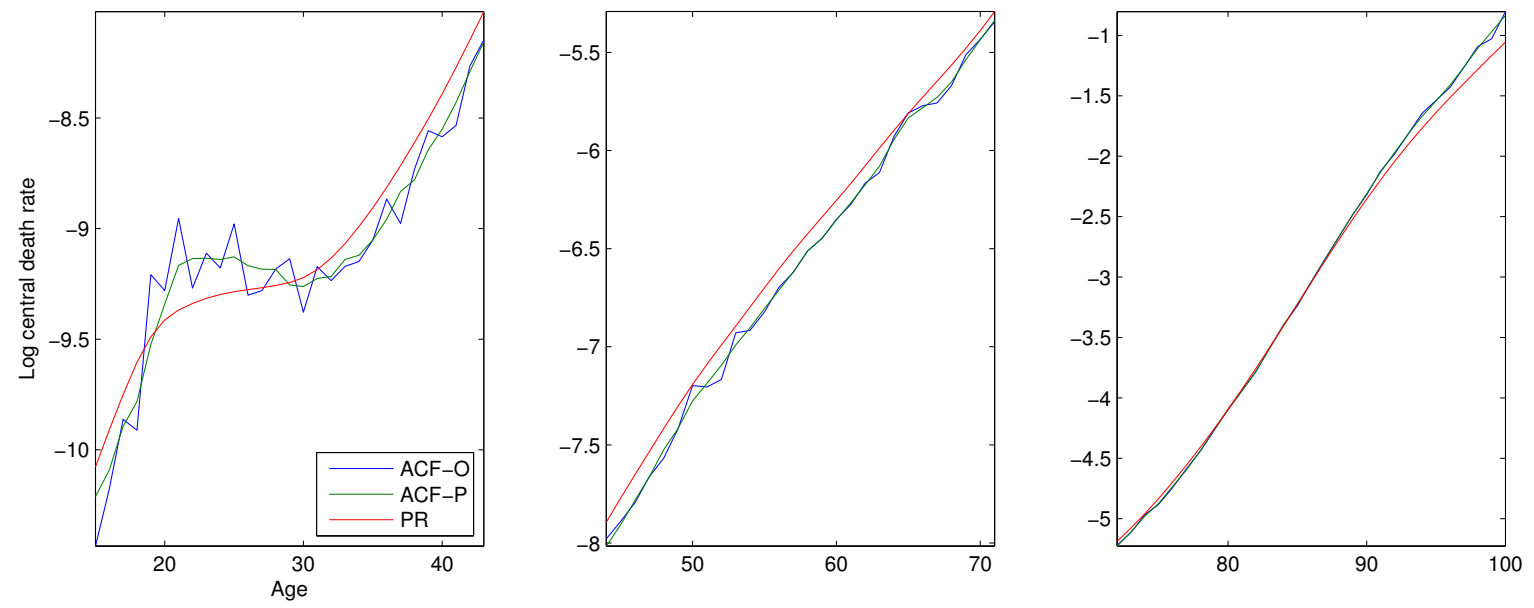

Figure 10: Forecasts of the log central death rates for Swedish females, 1, 10 and 60 year(s) beyond the forecast origin, generated from the ACF-O, ACF-P and PR models. 
modeled by a random walk with drift) while the deviations from $K_{t}$ are absorbed by $k_{t, 1}$ and $k_{t, 2}$ (furthered modeled by a first order autoregression). As we rewrite equation (5.2) further as

$$
\frac{m_{x, t, 1}}{m_{x, t, 2}}=\exp \left(a_{x, 1}-a_{x, 2}+b_{x, 1} k_{t, 1}-b_{x, 2} k_{t, 2}+\varepsilon_{x, t, 1}-\varepsilon_{x, t, 2}\right)
$$

and equation (5.3) as

$$
\frac{\tilde{m}_{x, t, 1}}{\tilde{m}_{x, t, 2}}=\exp \left(2 \mu_{x}^{r}+2 b_{x, 1} k_{t, 1}+2 b_{x, 2} k_{t, 2}+2 e_{x, t}^{r}\right)
$$

we can discern that both model structures generate coherent forecasts in the sense that the projected mortality ratio $\left(m_{x, t, 1} / m_{x, t, 2}\right.$ for the ACF model and $\tilde{m}_{x, t, 1} / \tilde{m}_{x, t, 2}$ for the PR model) reverts to a constant as $t$ increases, due to the fact that $e_{x, t}^{r}, \varepsilon_{x, t, 1}$ and $\varepsilon_{x, t, 2}$ have a zero mean while $k_{t, 1}$ and $k_{t, 2}$ are both mean-reverting. It should be emphasized, however, that the absolute difference, $m_{x, t, 1}-m_{x, t, 1}$ (for the ACF model) or $\tilde{m}_{x, t, 1}-\tilde{m}_{x, t, 2}$ (for the PR model), does change steadily with time as the magnitude of $K_{t}$ grows with $t$ increases.

Despite these similarities, there exist several fundamental differences between the two model structures. First, it is obvious that the quantities being modeled by the two model structures are not identical: equation (5.2) is built for crude central death rates $\left(m_{x, t, 1}\right.$ and $\left.m_{x, t, 2}\right)$ whereas equation (5.3) is constructed for pre-smoothed central death rates $\left(\tilde{m}_{x, t, 1}\right.$ and $\left.\tilde{m}_{x, t, 2}\right)$. Second, the population-specific characteristics for each population are driven by two stochastic factors $\left(k_{t, 1}\right.$ and $\left.k_{t, 2}\right)$ in the PR model but only one stochastic factor $\left(k_{t, 1}\right.$ or $\left.k_{t, 2}\right)$ in the ACF model. Third, while the ACF model permits asymmetric deviations from the common time trend, the PR model does not. Specifically, in the PR model, if a log central death rate for population 1 deviates from the common time trend by an amount of $b_{x, 1} k_{t, 1}+b_{x, 2} k_{t, 2}$, then the corresponding log central death rate for population 2 must change by the same amount in the opposite direction. ${ }^{8}$ It is noteworthy that even if the PR model is fitted to crude instead of smoothed death rates, it still cannot be regarded as a special case or a generalization of the ACF model. The PR model is equivalent to the ACF model only in very special circumstances (e.g., when $b_{x, 1}=-b_{x, 2}$ and $k_{t, 1}=k_{t, 2}$ in the ACF model), which are unlikely to hold. Finally, while no distributional assumption is required in fitting the PR model, a Poisson death count assumption is needed when estimating the ACF model with the penalized log-likelihood method. The requirement of a distributional assumption may be regarded as a drawback, because the assumed distribution may not be sufficiently realistic. For example, Li et al. (2009) demonstrated that in some situations, a negative binomial assumption is more appropriate than a Poisson assumption.

When estimating the model parameters, more weights may be given to the more recent observations. In this way, the resulting forecasts are likely to be more in line with the most recent

\footnotetext{
${ }^{8}$ This statement ignores the error terms $e_{x, t}^{p}, e_{x, t}^{r}, \varepsilon_{x, t, 1}$ and $\varepsilon_{x, t, 2}$, all of which have a zero mean and consequently have no impact on the resulting central forecasts. Further, the uncertainty surrounding the error terms does not grow with time, and therefore only contributes minimally to the overall forecast uncertainty when the forecast horizon is long.
} 
observed trends, and the model parameter estimates tend to be less sensitive to the beginning point of the calibration window. When estimating the PR model, Hyndman et al. (2013) assigned a weight of 0.05 to the latest observation, $0.05 \times(1-0.05)$ to the second latest observation, and so on. A similar strategy can be used when estimating the ACF models. If the weighted penalized least square estimation method is used, then the objective function would become

$$
\sum_{i=1}^{n_{p}} \sum_{x=x_{\min }}^{x_{\max }} \sum_{t=t_{\min }}^{t_{\max }} w_{t}\left(\ln m_{x, t, i}-a_{x, i}-B_{x} K_{t}-b_{x, i} k_{t, i}\right)^{2}+\vec{B}^{\prime} \mathbf{P}_{c} \vec{B}+\sum_{i=1}^{n_{p}} \vec{b}_{i}^{\prime} \mathbf{P}_{i} \vec{b}_{i},
$$

and if the weighted (penalized) log-likelihood estimation method is used, the log-likelihood would be changed to

$$
l=\sum_{i=1}^{n_{p}} \sum_{x=x_{\min }}^{x_{\max }} \sum_{t=t_{\min }}^{t_{\max }} w_{t}\left(d_{x, t, i}\left(a_{x, i}+B_{x} K_{t}+b_{x, i} k_{t, i}\right)-E_{x, t, i} \exp \left(a_{x, i}+B_{x} K_{t}+b_{x, i} k_{t, i}\right)\right),
$$

where $w_{t}$ represents the weight imposed on the observations made in year $t$. It should be noted, however, that it does not seem straightforward to optimize $w_{t}, t=t_{\min }, \ldots, t_{\max }$, on the basis of a rigorous statistical criterion. ${ }^{9}$

We may use ARFIMA rather than ARIMA processes to model $k_{1, t}$ and $k_{2, t}$ in both models. Compared to stationary ARIMA processes, ARFIMA processes allow $k_{1, t}, k_{2, t}$ and thus the mortality ratio $\left(m_{x, t, 1} / m_{x, t, 2}\right.$ for the ACF model and $\tilde{m}_{x, t, 1} / \tilde{m}_{x, t, 2}$ for the PR model) to revert to their long-term equilibria more slowly. Depending on the actual mortality dynamics, a slower convergence rate may lead to more accurate forecasts. However, as Hyndman et al. (2013) pointed out, stable estimation of ARFIMA processes requires a long data series, which means that ARFIMA processes may not be suitable for shorter data series such as our Data Set II. As a fact, when $k_{1, t}$ and $k_{2, t}$ from Data Set II are fitted to ARFIMA processes, the estimated values of $d$ are smaller than $10^{-4}$, suggesting that it would make little difference to the resulting forecasts if ARFIMA processes are used instead.

We conclude this section with a comment on the possibility of extending the ACF and PR models to incorporate cohort effects, which are found to be significant in populations such as England and Wales (see, e.g., Cairns et al., 2009). We may add cohort effects to the ACF model structure as follows:

$$
\ln \left(m_{x, t, i}\right)=a_{x, i}+B_{x} K_{t}+C_{x} \Gamma_{t-x}+b_{x, i} k_{t, i}+c_{x, i} \gamma_{t-x, i}+\varepsilon_{x, t, i}, \quad i=1,2
$$

where parameters $\Gamma_{t-x}$ and $\gamma_{t-x, i}$ capture cohort effects as they reflect the characteristics that are specific to the individuals who were born in year $t-x$, and parameters $C_{x}$ and $c_{x, i}$ represent the sensitivities to $\Gamma_{t-x}$ and $\gamma_{t-x, i}$ at age $x$, respectively. By modifying expressions (3.1), (3.6) and (3.7) accordingly, the extended ACF model can be estimated using penalized least squares

\footnotetext{
${ }^{9}$ Hyndman et al. (2013, p.275) acknowledged that the weight parameter of 0.05 they used may not be optimal at all ages and in all cases.
} 
or log-likelihood methods. In fact, similar extensions have been considered by Li et al. (2015). In principle, the PR model may also be generalized to capture cohort effects as follows:

$$
\ln \left(p_{x, t}\right)=\mu_{x}^{p}+\sum_{j=1}^{N_{p}} B_{j, x} K_{j, t}+\sum_{j=1}^{N_{p}^{\prime}} C_{j, x} \Gamma_{j, t-x}+e_{x, t}^{p}
$$

and

$$
\ln \left(r_{x, t}\right)=\mu_{x}^{r}+\sum_{j=1}^{N_{r}} b_{j, x} k_{j, t}+\sum_{j=1}^{N_{r}^{\prime}} c_{j, x} \gamma_{j, t-x}+e_{x, t}^{r},
$$

where $N_{p}^{\prime}$ and $N_{r}^{\prime}$ are positive integers, $\Gamma_{j, t-x}$ and $\gamma_{j, t-x}$ are parameters that capture cohort effects, and $C_{j, x}$ and $c_{j, x}$ measure the sensitivities to $\Gamma_{j, t-x}$ and $\gamma_{j, t-x}$ at age $x$, respectively. However, it is unclear to the authors as to how the extended PR model can be estimated using a principal component decomposition. Further research on the estimation of such a model is required.

\section{Concluding Remarks}

By synthesizing the work of $\mathrm{Li}$ and Lee (2005) and Delwarde et al. (2007), we contribute in this paper methods that permit us to generate forecasts of multi-population mortality with smooth patterns across ages. We illustrate the proposed methods with several sets of mortality data. It is found that the role of the roughness penalties imposed is the most important in forecasts for younger ages (over which the crude estimates are the most erratic) and also in forecasts for longer horizons (when the effect of the jaggedness in the crude estimates is much amplified).

It should be noted that the problem of erratic patterns may not be the only problem concerning the age-response parameters $B_{x}$ and $b_{x, i}$ in the ACF model. As pointed out by Booth et al. (2002), the pattern of $b_{x}$ in the (single-population) Lee-Carter model may vary as calibration window extends further to the past. The same problem may also apply to parameters $B_{x}$ and $b_{x, i}$ in the ACF model. One possible solution to weight the more recent observations heavier in the estimation procedure, as described in Section 5.4. Another possible solution is to identify an optimal calibration window on the basis of a statistical criterion ( $\mathrm{Li}$ and $\mathrm{Li}, 2017$ ). Further research warrants an investigation on how an optimal calibration window may be determined when multiple populations are modeled in tandem.

To our knowledge, this paper represents the first attempt to introduce within-estimation roughness penalties in the context of multi-population mortality forecasting. ${ }^{10}$ Building on this first attempt, future research may investigate how roughness penalties may be imposed in more sophisticated multi-population stochastic mortality models, including those that incorporate cohort

\footnotetext{
${ }^{10}$ Using an extended GLM model structure, Pitt et al. (2018) also developed a multi-population mortality model in which estimation and smoothing are performed in the same phase. Their work was published after this paper was completed and circulated among the academic community for peer review and comments.
} 
effects (Li et al., 2015; Yang et al., 2014). In terms of practical applications, it would be interesting to study the impact of the imposed roughness penalties on insurance pricing and mortality/longevity risk management.

\section{Acknowledgment}

This work is supported by research grants from NSERC Canada (Discovery Grant RGPIN-3560502013), and the Society of Actuaries Center of Actuarial Excellence Program.

\section{References}

Ahmadi, S. S. and J. S.-H. Li (2014). Coherent mortality forecasting with generalized linear models: A modified time-transformation approach. Insurance: Mathematics and Economics 59, 194-221.

Booth, H. (2006). Demographic forecasting: 1980 to 2005 in review. International Journal of Forecasting 22(3), 547-581.

Booth, H., R. Hyndman, L. Tickle, and P. de Jong (2006). Lee-Carter mortality forecasting: A multicountry comparison of variants and extensions. Demographic Research 15(9), 289-310.

Booth, H., J. Maindonald, and L. Smith (2002). Applying Lee-Carter under conditions of variable mortality decline. Population Studies 56(3), 325-336.

Booth, H., L. Tickle, and L. Smith (2005). Evaluation of the variants of the Lee-Carter method of forecasting mortality: A multi-country comparison. New Zealand Population Review 31(1), 13-34.

Cairns, A. J., D. Blake, K. Dowd, G. D. Coughlan, D. Epstein, A. Ong, and I. Balevich (2009). A quantitative comparison of stochastic mortality models using data from England and Wales and the United States. North American Actuarial Journal 13(1), 1-35.

Chan, W.-S., J. S.-H. Li, K. Q. Zhou, and R. Zhou (2016). Towards a large and liquid longevity market: A graphical population basis risk metric. The Geneva Papers on Risk and Insurance-Issues and Practice 41(1), 118-127.

Currie, I. D., M. Durban, and P. H. Eilers (2004). Smoothing and forecasting mortality rates. Statistical Modelling 4(4), 279-298.

De Jong, P. and L. Tickle (2006). Extending Lee-Carter mortality forecasting. Mathematical Population Studies 13(1), 1-18.

Delwarde, A., M. Denuit, and P. Eilers (2007). Smoothing the Lee-Carter and Poisson log-bilinear models for mortality forecasting a penalized log-likelihood approach. Statistical Modelling 7(1), 29-48.

Hunt, A. and D. Blake (2015). Modelling longevity bonds: Analysing the Swiss Re Kortis bond. Insurance: Mathematics and Economics 63, $12-29$. 
Hyndman, R. J., H. Booth, and F. Yasmeen (2013). Coherent mortality forecasting: The product-ratio method with functional time series models. Demography 50(1), 261-283.

Hyndman, R. J. and M. S. Ullah (2007). Robust forecasting of mortality and fertility rates: A functional data approach. Computational Statistics $\&$ Data Analysis 51(10), 4942-4956.

Hyndman, R. J., with contributions from Heather Booth, L. Tickle, and J. Maindonald (2014). demography: Forecasting mortality, fertility, migration and population data. $\mathrm{R}$ package version 1.18 .

Kleinow, T. (2015). A common age effect model for the mortality of multiple populations. Insurance: Mathematics and Economics 63, $147-152$.

Lee, R. D. and L. R. Carter (1992). Modeling and forecasting US mortality. Journal of the American statistical association 87(419), 659-671.

Li, H. and J. S.-H. Li (2017). Optimizing the Lee-Carter approach in the presence of structural changes in time and age patterns of mortality improvements. Demography 54(3), 1073-1095.

Li, J. (2013). A Poisson common factor model for projecting mortality and life expectancy jointly for females and males. Population Studies 67(1), 111-126.

Li, J., L. Tickle, C. Tan, and J. S.-H. Li (2017). Assessing basis risk for longevity transactions-phase 2, Macquarie University, Institute and Faculty of Actuaries, December 2017.

Li, J., L. Tickle, C. Tan, and J. S.-H. Li (2018). Assessing basis risk in index-based longevity swap transactions. Annals of Actuarial Science, to appear.

Li, J. S.-H., W.-S. Chan, and R. Zhou (2017). Semi-coherent multi-population mortality modeling: The impact on longevity risk securitization. Journal of Risk and Insurance 84(3), 1025-1065.

Li, J. S.-H. and M. R. Hardy (2011). Measuring basis risk in longevity hedges. North American Actuarial Journal 15(2), 177-200.

Li, J. S.-H., M. R. Hardy, and K. S. Tan (2009). Uncertainty in mortality forecasting: An extension to the classical Lee-Carter approach. ASTIN Bulletin 39(01), 137-164.

Li, J. S.-H., R. Zhou, and M. Hardy (2015). A step-by-step guide to building two-population stochastic mortality models. Insurance: Mathematics and Economics 63, 121-134.

Li, N. and R. Lee (2005). Coherent mortality forecasts for a group of populations: An extension of the Lee-Carter method. Demography 42(3), 575-594.

Liu, Y. and J. S.-H. Li (2016). Its all in the hidden states: A longevity hedging strategy with an explicit measure of population basis risk. Insurance: Mathematics and Economics 70, 301-319.

Pitt, D., J. Li, and T. K. Lim (2018). Smoothing Poisson common factor model for projecting mortality jointly for both sexes. ASTIN Bulletin: The Journal of the IAA, 1-33.

Plat, R. (2009). Stochastic portfolio specific mortality and the quantification of mortality basis risk. Insurance: Mathematics and Economics 45(1), 123 - 132. 
Renshaw, A. E. and S. Haberman (2003). On the forecasting of mortality reduction factors. Insurance: Mathematics and Economics 32(3), 379-401.

Wan, C. and L. Bertschi (2015). Swiss coherent mortality model as a basis for developing longevity de-risking solutions for Swiss pension funds: A practical approach. Insurance: Mathematics and Economics $63,66-75$.

Wood, S. (1994). Monotonic smoothing splines fitted by cross validation. SIAM Journal on Scientific Computing 15(5), 1126-1133.

Yang, B., J. Li, and U. Balasooriya (2014). Cohort extensions of the Poisson common factor model for modelling both genders jointly. Scandinavian Actuarial Journal (ahead-of-print), 1-20.

Zhou, K. Q. and J. S.-H. Li (2017). Dynamic longevity hedging in the presence of population basis risk: A feasibility analysis from technical and economic perspectives. Journal of Risk and Insurance 84(S1), 417-437. 